\title{
Random Attractors for Stochastic Ginzburg-Landau Equation on Unbounded Domains
}

\author{
Qiuying Lu, ${ }^{1}$ Guifeng Deng, ${ }^{2}$ and Weipeng Zhang ${ }^{3}$ \\ ${ }^{1}$ Department of Mathematics, Zhejiang Sci-Tech University, Hangzhou, Zhejiang 310018, China \\ ${ }^{2}$ School of Mathematics and Information Science, Shanghai Lixin University of Commerce, Shanghai 201620, China \\ ${ }^{3}$ School of Mathematics and Statistics, Northeast Normal University, Changchun, Jilin 130024, China
}

Correspondence should be addressed to Qiuying Lu; qiuyinglu@163.com

Received 18 February 2014; Revised 26 April 2014; Accepted 3 May 2014; Published 1 June 2014

Academic Editor: Milan Pokorny

Copyright (C) 2014 Qiuying Lu et al. This is an open access article distributed under the Creative Commons Attribution License, which permits unrestricted use, distribution, and reproduction in any medium, provided the original work is properly cited.

We prove the existence of a pullback attractor in $\mathbb{L}^{2}\left(\mathbb{R}^{n}\right)$ for the stochastic Ginzburg-Landau equation with additive noise on the entire $n$-dimensional space $\mathbb{R}^{n}$. We show that the stochastic Ginzburg-Landau equation with additive noise can be recast as a random dynamical system. We demonstrate that the system possesses a unique $\mathscr{D}$-random attractor, for which the asymptotic compactness is established by the method of uniform estimates on the tails of its solutions.

\section{Introduction}

In this paper, we study the following stochastic GinzburgLandau equation with additive noise defined in the entire space $\mathbb{R}^{n}$ :

$$
\begin{aligned}
\mathrm{d} u= & (\lambda+i \mu) \Delta u d t-(\kappa+i \beta)|u|^{2} u d t \\
& -\gamma u d t+\sum_{j=1}^{m} \varphi_{j} \mathrm{~d} \omega_{j}(t),
\end{aligned}
$$

with the initial condition

$$
u(x, 0)=u_{0}(x), \quad x \in \mathbb{R}^{n},
$$

where $\lambda, \mu, \kappa, \beta, \gamma$ are real coefficients, with $\lambda>0, \kappa>$ $0, \gamma>0$, and $\varphi_{j} \in H^{2}\left(\mathbb{R}^{n}\right) \cap W^{2,4}\left(\mathbb{R}^{n}\right), j=1, \ldots, m$, being time independent defined on $\mathbb{R}^{n}$ and $\left\{\omega_{j}\right\}_{j=1}^{m}$ being independent two-sided real-valued Wiener processes on a complete probability space $(\Omega, \mathscr{F}, P)$. Our aim is to study its long time behavior defined in the entire space $\mathbb{R}^{n}$.

Attractors are quite well investigated to describe the long time behavior of the deterministic equations (see, e.g., [1-7]). Recently, the concept of random attractors, which is in fact compact invariant set, was introduced to stochastic dynamical systems from the theory of attractors for deterministic equations in [8-10]. The existence of such random attractors for the Ginzburg-Landau equation perturbed by additive white noise and multiplicative white noise on bounded domains has been investigated, respectively, in $[11,12]$.

However, for unbounded domains, we cannot guarantee the compactness of solutions by the standard method since the Sobolev embeddings are no longer compact. Hence, to prove the existence of an attractor, we have to first overcome this difficulty. For deterministic equations, this difficulty has been overcome by employing the energy equation approach, introduced in $[13,14]$, and then used by others to prove the asymptotic compactness of deterministic equations in unbounded domains (see, e.g., [15-22]). In this paper, we prove the existence of a random attractor for the stochastic Ginzburg-Landau equation (1), defined on the unbounded domain $\mathbb{R}^{n}$ with the help of tail estimates method, which was firstly established in [23] to the case of stochastic dissipative PDEs.

For the mathematical setting, we introduce complex Sobolev spaces. In general, we denote by $\mathbb{X}, \mathbb{Y}, \ldots$ the complexified space of a function space $X, Y, \ldots$. For example, $\mathbb{L}^{2}\left(\mathbb{R}^{n}\right)$ is the complexified space of $L^{2}\left(\mathbb{R}^{n}\right)$. Denote by $(\cdot, \cdot)$ and $\|\cdot\|_{L^{2}}$ the scalar product and the norm in either $L^{2}\left(\mathbb{R}^{n}\right)$ or $\mathbb{L}^{2}\left(\mathbb{R}^{n}\right)$. So, if $u \in \mathbb{L}^{2}\left(\mathbb{R}^{n}\right)$, then $u=\left\{u_{1}, u_{2}\right\}, u_{j} \in L^{2}\left(\mathbb{R}^{n}\right)$, $j=1,2$, and

$$
\|u\|_{L^{2}}=\left\{\left\|u_{1}\right\|_{L^{2}}^{2}+\left\|u_{2}\right\|_{L^{2}}^{2}\right\}^{1 / 2} .
$$


If $u=u_{1}+i u_{2}, v=v_{1}+i v_{2}$ are in $\mathbb{L}^{2}\left(\mathbb{R}^{n}\right)$,

$$
(u, v)=\left\{\left(u_{1}, v_{1}\right)+\left(u_{2}, v_{2}\right)\right\}+i\left\{\left(u_{2}, v_{1}\right)-\left(u_{1}, v_{2}\right)\right\} .
$$

We use letter $c>0$ to denote any positive constant which may change its value from line to line or even in the same line when necessary.

The whole paper is organized as follows. In Section 2, we first recall some definitions and propositions on random attractors for random dynamical systems (RDS). And then, by Ornstein-Uhlenbeck process, we obtain the continuous RDS $\phi$ associated with the stochastic Ginzburg-Landau equation (1). In Section 3, we concentrate to get the uniform estimate on the far-field values of the solution as $t \longrightarrow \infty$ and thus to further establish the asymptotic compactness of the solution operator $\phi$. Then, we can exhibit our main result in the following theorem.

Theorem 1. The random dynamical system $\phi$ of stochastic Ginzburg-Landau equation with additive noise has a unique D-random attractor in $\mathbb{L}^{2}\left(\mathbb{R}^{n}\right)$ provided that $\sqrt{3} \kappa \geq|\beta|$.

\section{RDS Associated with the Stochastic Ginzburg-Landau Equation on $\mathbb{R}^{n}$}

2.1. Preliminaries on $R D S$. We first recall some definitions. For more details, one can refer to $[8,10,24-26]$.

Definition 2. Let $(\Omega, \mathscr{F}, P)$ be a probability space and $\left\{\theta_{t}\right.$ : $\Omega \longrightarrow \Omega, t \in \mathbb{R}\}$ a family of measures preserving transformation such that $(t, \omega) \longrightarrow \theta_{t} \omega$ is measurable, $\theta_{0}=$ id, and $\theta_{s+t}=\theta_{t} \circ \theta_{s}$, for all $s, t \in \mathbb{R}$, and then the flow $\theta_{t}$ together with the corresponding probability space $\left(\Omega, \mathscr{F}, P,\left(\theta_{t}\right)_{t \in \mathbb{R}}\right)$ is called a metric dynamical system.

For Wiener process $\omega_{j}$ in (1), we consider the probability space $(\Omega, \mathscr{F}, P)$, where

$$
\Omega=\left\{\omega=\left(\omega_{1}, \omega_{2}, \ldots, \omega_{m}\right) \in C\left(\mathbb{R}, \mathbb{R}^{m}\right), \omega(0)=0\right\},
$$

$\mathscr{F}$ is the Borel $\sigma$-algebra induced by the compact-open topology of $\Omega$, and $P$ is the corresponding Wiener measure on $(\Omega, \mathscr{F})$. The time shift is simply defined by

$$
\theta_{t} \omega(s)=\omega(s+t)-\omega(s), \quad t, s \in \mathbb{R}, \omega \in \Omega .
$$

Then $\left(\Omega, \mathscr{F}, P,\left(\theta_{t}\right)_{t \in R}\right)$ is a metric dynamical system.

Definition 3. A continuous random dynamical system (RDS) on $X$ over a metric dynamical system $\left(\Omega, \mathscr{F}, P,\left(\theta_{t}\right)_{t \in R}\right)$ is a mapping:

$$
\phi: \mathbb{R}^{+} \times \Omega \times X \longrightarrow X, \quad(t, \omega, x) \longrightarrow \phi(t, \omega, x),
$$

which is $\left(\mathscr{B}\left(\mathbb{R}^{+}\right) \times \mathscr{F} \times \mathscr{B}(X), \mathscr{B}(X)\right)$-measurable such that, for $P$-a.e. $\omega \in \Omega$,

(i) $\phi(0, \omega, \cdot)$ is the identity on $X$;

(ii) $\phi(t+s, \omega, \cdot)=\phi\left(t, \theta_{s} \omega, \cdot\right) \circ \phi(s, \omega, \cdot)$ for all $t, s \in \mathbb{R}^{+}$;

(iii) $\phi(t, \omega, \cdot): X \longrightarrow X$ is continuous for all $t \in \mathbb{R}^{+}$.
Hereafter, we always assume that $\phi$ is a continuous RDS on $X$ over $\left(\Omega, \mathscr{F}, P,\left(\theta_{t}\right)_{t \in R}\right)$.

Definition 4. A random variable $R: \Omega \longrightarrow(0, \infty)$ is called tempered with respect to the dynamical system $\theta$ if, for the associated stationary stochastic process $t \longrightarrow R\left(\theta_{t^{*}}\right)$, the invariant set for which

$$
\lim _{t \rightarrow \pm \infty} \frac{1}{t} \log R\left(\theta_{t} \omega\right)=0
$$

$(t \longrightarrow-\infty$ applies only to two-sided time) has full $P$-measure.

Definition 5. A random bounded set $\{B(\omega)\}_{\omega \in \Omega}$ of $X$ is called tempered with respect to $\left(\theta_{t}\right)_{t \in \mathbb{R}}$ if, for $P$-a.e. $\omega \in \Omega$,

$$
\lim _{t \rightarrow \infty} e^{-\varepsilon t} d\left(B\left(\theta_{-t} \omega\right)\right)=0 \quad \forall \varepsilon>0,
$$

where $d(B)=\sup _{x \in B}\|x\|_{X}$.

Definition 6. Let $\mathscr{D}$ be a collection of random subsets of $X$ and $\{K(\omega)\}_{\omega \in \Omega} \in \mathscr{D}$. Then $\{K(\omega)\}_{\omega \in \Omega}$ is called a random absorbing set for $\phi$ in $\mathscr{D}$ if, for every $B \in \mathscr{D}$ and $P$-a.e. $\omega \in \Omega$, there exists $t_{B}(\omega)>0$ such that

$$
\phi\left(t, \theta_{-t} \omega, B\left(\theta_{-t} \omega\right)\right) \subseteq K(\omega) \quad \forall t \geq t_{B}(\omega) .
$$

Definition 7. Let $\mathscr{D}$ be a collection of random subsets of $X$. Then $\phi$ is said to be $\mathscr{D}$-pullback asymptotically compact in $X$ if, for $P$-a.e. $\omega \in \Omega,\left\{\phi\left(t_{n}, \theta_{-t_{n}} \omega, x_{n}\right)\right\}_{n=1}^{\infty}$ has a convergent subsequence in $X$ whenever $t_{n} \longrightarrow \infty$, and $x_{n} \in B\left(\theta_{-t_{n}} \omega\right)$ with $\{B(\omega)\}_{\omega \in \Omega} \in \mathscr{D}$.

Definition 8. Let $\mathscr{D}$ be a collection of random subsets of $X$. Then a random set $\{\mathscr{A}(\omega)\}_{\omega \in \Omega}$ of $X$ is called a $\mathscr{D}$-random attractor (or $\mathscr{D}$-pullback attractor) for $\phi$ if the following conditions are satisfied: for $P$-a.e. $\omega \in \Omega$,

(i) $\mathscr{A}(\omega)$ is compact, and $\omega \longrightarrow d(x, \mathscr{A}(\omega))$ is measurable for every $x \in X$;

(ii) $\mathscr{A}(\omega)$ is invariant; that is,

$$
\phi(t, \omega, \mathscr{A}(\omega))=\mathscr{A}\left(\theta_{t} \omega\right) \quad \forall t \geq 0 ;
$$

(iii) $\mathscr{A}(\omega)$ attracts every set in $\mathscr{D}$; that is, for every $B=$ $\{B(\omega)\}_{\omega \in \Omega} \in \mathscr{D}$,

$$
\lim _{t \rightarrow \infty} d\left(\phi\left(t, \theta_{-t} \omega, B\left(\theta_{-t} \omega\right)\right), \mathscr{A}(\omega)\right)=0
$$

where $d$ is the Hausdorff semimetric given by $d(Y, Z)=$ $\sup _{y \in Y} \inf _{z \in Z}\|y-z\|_{X}$ for any $Y \subseteq X$ and $Z \subseteq X$.

Proposition 9 (see $[10,25])$. Let $\mathscr{D}$ be an inclusion-closed collection of random subsets of $X$ and $\phi$ a continuous RDS on $X$ over $\left(\Omega, \mathscr{F}, P,\left(\theta_{t}\right)_{t \in R}\right)$. Suppose that $\{K(\omega)\}_{\omega \in \Omega}$ is a closed random absorbing set for $\phi$ in $\mathscr{D}$ and $\phi$ is $\mathscr{D}$-pullback asymptotically compact in $X$. Then $\phi$ has a unique $D$-random attractor $\{\mathscr{A}(\omega)\}_{\omega \in \Omega}$ which is given by

$$
\mathscr{A}(\omega)=\bigcap_{\tau \geq 0} \overline{\bigcup_{t \geq \tau} \phi\left(t, \theta_{-t} \omega, K\left(\theta_{-t} \omega\right)\right)} .
$$


Remark 10. A collection $\mathscr{D}$ of random subsets is called inclusion closed if, whenever $\{E(\omega)\}_{\omega \in \Omega}$ is an arbitrary random set and $\{F(\omega)\}_{\omega \in \Omega}$ is in $\mathscr{D}$ with $E(\omega) \subset F(\omega)$, for all $\omega \in \Omega$, $\{E(\omega)\}_{\omega \in \Omega}$ must belong to $\mathscr{D}$.

2.2. RDS Associated with the Stochastic Ginzburg-Landau Equation on $\mathbb{R}^{n}$. Denote $z(t)=z\left(\theta_{t} \omega\right)=\sum_{j=1}^{m} \varphi_{j} z_{j}\left(\theta_{t} \omega_{j}\right)$, where

$$
z_{j}(t)=z_{j}\left(\theta_{t} \omega_{j}\right)=\int_{-\infty}^{t} e^{\gamma(s-t)} \mathrm{d} \omega_{j}(s), \quad t \in \mathbb{R},
$$

satisfies the one-dimensional Ornstein-Uhlenbeck equation

$$
\mathrm{d} z_{j}=-\gamma z_{j} d t+\mathrm{d} \omega_{j}(t)
$$

Since the random variable $\left|z_{j}\left(\omega_{j}\right)\right|$ is tempered and $\left|z_{j}\left(\theta_{t} \omega_{j}\right)\right|$ is $P$-a.e. continuous, there exists a tempered function $r(\omega)>$ 0 such that

$$
\sum_{j=1}^{m}\left(\left|z_{j}\left(\omega_{j}\right)\right|^{2}+\left|z_{j}\left(\omega_{j}\right)\right|^{4}\right) \leq r(\omega)
$$

where $r(\omega)$ satisfies, for $P$-a.e. $\omega \in \Omega$,

$$
r\left(\theta_{t} \omega\right) \leq e^{(\gamma / 2)|t|} r(\omega), \quad t \in \mathbb{R},
$$

thanks to Proposition 4.3.3 in [24]. From (16) to (17), we get, for $P$-a.e. $\omega \in \Omega$,

$$
\sum_{j=1}^{m}\left(\left|z_{j}\left(\theta_{t} \omega_{j}\right)\right|^{2}+\left|z_{j}\left(\theta_{t} \omega_{j}\right)\right|^{4}\right) \leq e^{(\gamma / 2)|t|} r(\omega), \quad t \in \mathbb{R} .
$$

Introduce the transformation

$$
v(t)=u(t)-z\left(\theta_{t} \omega\right)
$$

where $u$ is the solution of (1)-(2); then $v$ should satisfy

$$
\begin{aligned}
\frac{\partial v}{\partial t}= & (\lambda+i \mu) \Delta v-(\kappa+i \beta)|v+z|^{2}(v+z) \\
& -\gamma v+(\lambda+i \mu) \Delta z .
\end{aligned}
$$

Similar to the procedure in [23], we can obtain that (20) has a unique solution $v\left(t, \omega, v_{0}\right)$ with $v\left(0, \omega, v_{0}\right)=v_{0}$, which is continuous with respect to $v_{0}$ in $\mathbb{L}^{2}\left(\mathbb{R}^{n}\right)$. Let $u\left(t, \omega, u_{0}\right)=$ $v\left(t, \omega, u_{0}-z(\omega)\right)+z\left(\theta_{t} \omega\right)$, and then $u$ is the solution of (1)-(2). Define $\phi: \mathbb{R}^{+} \times \Omega \times \mathbb{L}^{2}\left(\mathbb{R}^{n}\right) \longrightarrow \mathbb{L}^{2}\left(\mathbb{R}^{n}\right)$ by

$$
\phi\left(t, \omega, u_{0}\right)=u\left(t, \omega, u_{0}\right)=v\left(t, \omega, u_{0}-z(\omega)\right)+z\left(\theta_{t} \omega\right),
$$

for all $\left(t, \omega, u_{0}\right) \in \mathbb{R}^{+} \times \Omega \times \mathbb{L}^{2}\left(\mathbb{R}^{n}\right)$. Then, we can claim that $\phi$ is a continuous random dynamical system associated with the stochastic Ginzburg-Landau equation on $\mathbb{R}^{n}$.

\section{Existence of Random Attractors}

In the following paper, we always assume that $\mathscr{D}$ is the collection of all tempered subsets of $\mathbb{L}^{2}\left(\mathbb{R}^{n}\right)$ with respect to $\left(\Omega, \mathscr{F}, P,\left(\theta_{t}\right)_{t \in R}\right)$. And then we are devoted to prove that $\phi$ has a random absorbing set in $\mathscr{D}$, and it is also $\mathscr{D}$-pullback asymptotically compact.

Proposition 11. There exists $\{K(\omega)\}_{\omega \in \Omega} \in \mathscr{D}$ such that $\{K(\omega)\}_{\omega \in \Omega}$ is a random absorbing set for $\phi$ in $\mathscr{D}$. Precisely, for any $B=\{B(\omega)\}_{\omega \in \Omega} \in \mathscr{D}$ and $P$-a.e. $\omega \in \Omega$, there is $t_{B}(\omega)>0$ such that

$$
\phi\left(t, \theta_{-t} \omega, B\left(\theta_{-t} \omega\right)\right) \subseteq K(\omega) \quad \forall t \geq t_{B}(\omega)
$$

Proof. By multiplying (20) by $\bar{v}$, integrating over $\mathbb{R}^{n}$, and taking the real part, we get

$$
\begin{aligned}
\frac{1}{2} \frac{d}{d t}\|v\|^{2}= & \operatorname{Re}(\lambda+i \mu)(\Delta v, \bar{v}) \\
& -\operatorname{Re}(\kappa+i \beta)\left(|v+z|^{2}(v+z), \bar{v}\right) \\
& -\gamma\|v\|^{2}+\operatorname{Re}(\lambda+i \mu)\left(\Delta z\left(\theta_{t} \omega\right), \bar{v}\right) .
\end{aligned}
$$

Here

$$
\begin{gathered}
\operatorname{Re}(\lambda+i \mu)(\Delta v, \bar{v})=-\lambda\|\nabla v\|^{2}, \\
-\operatorname{Re}(\kappa+i \beta)\left(|v+z|^{2}(v+z), \bar{v}\right) \\
=-\operatorname{Re}(\kappa+i \beta)\left(|v+z|^{2}(v+z), \overline{v+z}\right) \\
+\operatorname{Re}(\kappa+i \beta)\left(|v+z|^{2}(v+z), \bar{z}\right) \\
=-\kappa\|u\|_{4}^{4}+\int_{\mathbb{R}^{n}}|\kappa+i \beta| \cdot|u|^{3}|z| \mathrm{d} x \\
\leq-\kappa\|u\|_{4}^{4}+\frac{1}{2} \kappa\|u\|_{4}^{4}+\frac{27\left(\kappa^{2}+\beta^{2}\right)^{2}}{32 \kappa^{3}}\|z\|_{4}^{4} \\
\leq-\frac{1}{2} \kappa\|u\|_{4}^{4}+\frac{27\left(\kappa^{2}+\beta^{2}\right)^{2}}{32 \kappa^{3}}\|z\|_{4}^{4}, \\
\operatorname{Re}(\lambda+i \mu)\left(\Delta z\left(\theta_{t} \omega\right), \bar{v}\right) \\
\leq \int_{\mathbb{R}^{n}}|\lambda+i \mu| \cdot\left|\nabla z\left(\theta_{t} \omega\right)\right||\nabla v| \mathrm{d} x \\
\leq \frac{\lambda}{2}\|\nabla v\|^{2}+\frac{\lambda^{2}+\mu^{2}}{2 \lambda}\|\nabla z\|^{2} .
\end{gathered}
$$

From (23) to (24),

$$
\begin{aligned}
\frac{d}{d t}\|v\|^{2}+ & \lambda\|\nabla v\|^{2}+2 \gamma\|v\|^{2}+\kappa\|u\|_{4}^{4} \\
\leq & \frac{27\left(\kappa^{2}+\beta^{2}\right)^{2}}{16 \kappa^{3}}\|z\|_{4}^{4}+\frac{\lambda^{2}+\mu^{2}}{\lambda}\|\nabla z\|^{2} .
\end{aligned}
$$


We can see that the right-hand side of (25) can be bounded by

$$
c \cdot \sum_{j=1}^{m}\left(\left|z_{j}\left(\theta_{t} \omega_{j}\right)\right|^{2}+\left|z_{j}\left(\theta_{t} \omega_{j}\right)\right|^{4}\right) \triangleq h\left(\theta_{t} \omega\right),
$$

since $z\left(\theta_{t} \omega\right)=\sum_{j=1}^{m} \varphi_{j} z_{j}\left(\theta_{t} \omega_{j}\right)$, where $\varphi_{j} \in H^{2}\left(\mathbb{R}^{n}\right) \cap$ $W^{2,4}\left(\mathbb{R}^{n}\right)$.

So, for $\forall t \geq 0$,

$$
\frac{d}{d t}\|v\|^{2}+\gamma\|v\|^{2} \leq \frac{d}{d t}\|v\|^{2}+2 \gamma\|v\|^{2} \leq h\left(\theta_{t} \omega\right),
$$

which leads to

$$
\begin{aligned}
\left\|v\left(t, \omega, v_{0}(\omega)\right)\right\|^{2} \leq & e^{-\gamma t}\left\|v_{0}(\omega)\right\|^{2} \\
& +\int_{0}^{t} e^{\gamma(s-t)} h\left(\theta_{s} \omega\right) \mathrm{d} s, \quad \forall t \geq 0,
\end{aligned}
$$

by multiplying (27) by $e^{\gamma t}$ and integrating from 0 to $t$.

By replacing $\omega$ by $\theta_{-t} \omega$, we derive from (18) and (28) that, for all $t \geq 0$,

$$
\begin{aligned}
& \left\|v\left(t, \theta_{-t} \omega, v_{0}\left(\theta_{-t} \omega\right)\right)\right\|^{2} \\
& \leq e^{-\gamma t}\left\|v_{0}\left(\theta_{-t} \omega\right)\right\|^{2}+\int_{0}^{t} e^{\gamma(s-t)} h\left(\theta_{s-t} \omega\right) \mathrm{d} s \\
& \leq e^{-\gamma t}\left\|v_{0}\left(\theta_{-t} \omega\right)\right\|^{2}+\int_{-t}^{0} e^{\gamma \tau} h\left(\theta_{\tau} \omega\right) \mathrm{d} \tau, \\
& \leq e^{-\gamma t}\left\|v_{0}\left(\theta_{-t} \omega\right)\right\|^{2}+c \cdot \int_{-t}^{0} e^{\gamma \tau} e^{-(\gamma / 2) \tau} r(\omega) \mathrm{d} \tau, \\
& \leq e^{-\gamma t}\left\|v_{0}\left(\theta_{-t} \omega\right)\right\|^{2}+c \cdot \frac{1}{\gamma} r(\omega) .
\end{aligned}
$$

By replacing $\omega$ by $\theta_{-t} \omega$ in (21), one has $\phi\left(t, \theta_{-t} \omega, u_{0}\left(\theta_{-t} \omega\right)\right)=$ $v\left(t, \theta_{-t} \omega, u_{0}\left(\theta_{-t} \omega\right)-z\left(\theta_{-t} \omega\right)\right)+z(\omega)$. Thereafter,

$$
\begin{aligned}
\| \phi( & \left.t, \theta_{-t} \omega, u_{0}\left(\theta_{-t} \omega\right)\right) \|^{2} \\
= & \left\|v\left(t, \theta_{-t} \omega, u_{0}\left(\theta_{-t} \omega\right)-z\left(\theta_{-t} \omega\right)\right)+z(\omega)\right\|^{2} \\
\leq & 2\left\|v\left(t, \theta_{-t} \omega, u_{0}\left(\theta_{-t} \omega\right)-z\left(\theta_{-t} \omega\right)\right)\right\|^{2}+2\|z(\omega)\|^{2} \\
\leq & 2 e^{-\gamma t}\left\|u_{0}\left(\theta_{-t} \omega\right)-z\left(\theta_{-t} \omega\right)\right\|^{2} \\
& +\frac{2 c}{\gamma} r(\omega)+2\|z(\omega)\|^{2} \\
\leq & 4 e^{-\gamma t}\left(\left\|u_{0}\left(\theta_{-t} \omega\right)\right\|^{2}+\left\|z\left(\theta_{-t} \omega\right)\right\|^{2}\right) \\
& +\frac{2 c}{\gamma} r(\omega)+2\|z(\omega)\|^{2} .
\end{aligned}
$$

Recall that both the random variable $\|z(\omega)\|^{2}$ and the random bounded set $\{B(\omega)\}_{\omega \in \Omega} \in \mathscr{D}$ are tempered. Then, for any $u_{0}\left(\theta_{-t} \omega\right) \in B\left(\theta_{-t} \omega\right)$, there exists $t_{B}(\omega)>0$ such that, for all $t>t_{B}(\omega)$

$$
\begin{aligned}
4 e^{-\gamma t} & \left(\left\|u_{0}\left(\theta_{-t} \omega\right)\right\|^{2}+\left\|z\left(\theta_{-t} \omega\right)\right\|^{2}\right) \\
& =4\left[\left(e^{-(\gamma / 2) t}\left\|u_{0}\left(\theta_{-t} \omega\right)\right\|\right)^{2}+\left(e^{-(\gamma / 2) t}\left\|z\left(\theta_{-t} \omega\right)\right\|\right)^{2}\right] \\
& \leq \frac{2 c}{\gamma} r(\omega) .
\end{aligned}
$$

So far, for all $t>t_{B}(\omega)$,

$$
\left\|\phi\left(t, \theta_{-t} \omega, u_{0}\left(\theta_{-t} \omega\right)\right)\right\|^{2} \leq \frac{4 c}{\gamma} r(\omega)+2\|z(\omega)\|^{2} .
$$

Select

$$
K(\omega)=\left\{u \in \mathbb{L}^{2}\left(\mathbb{R}^{n}\right):\|u\|^{2} \leq \frac{4 c}{\gamma} r(\omega)+2\|z(\omega)\|^{2}\right\} ;
$$

then $\{K(\omega)\}_{\omega \in \Omega} \in \mathscr{D}$ is a random absorbing set for $\phi$ in $\mathscr{D}$.

The proof is completed.

Lemma 12. Let $B=\{B(\omega)\}_{\omega \in \Omega} \in \mathscr{D}$ and $u_{0}(\omega) \in B(\omega)$, and then, for any $T_{1} \geq 0$ and $P$-a.e. $\omega \in \Omega$, that is, the two inequalities of (34) hold true for the solution $u\left(t, \omega, u_{0}(\omega)\right)$ of (1)-(2) and $v\left(t, \omega, v_{0}(\omega)\right)$ of (20) with $v_{0}(\omega)=u_{0}(\omega)-z(\omega)$, $t \geq T_{1}$, such that

$$
\begin{aligned}
& \int_{T_{1}}^{t} e^{\gamma(s-t)}\left\|u\left(s, \theta_{-t} \omega, u_{0}\left(\theta_{-t} \omega\right)\right)\right\|_{4}^{4} d s \\
& \leq \frac{1}{\kappa} e^{-\gamma t}\left\|v_{0}\left(\theta_{-t} \omega\right)\right\|^{2}+\frac{2 c}{\gamma \kappa} \cdot r(\omega), \\
& \int_{T_{1}}^{t} e^{\gamma(s-t)}\left\|\nabla v\left(s, \theta_{-t} \omega, v_{0}\left(\theta_{-t} \omega\right)\right)\right\|^{2} d s \\
& \leq \frac{1}{\lambda} e^{-\gamma t}\left\|v_{0}\left(\theta_{-t} \omega\right)\right\|^{2}+\frac{2 c}{\gamma \lambda} \cdot r(\omega) .
\end{aligned}
$$

Proof. Fix $T_{1} \geq 0$, and then replace $t$ by $T_{1}$ and $\omega$ by $\theta_{-t} \omega$ in (28); we then obtain

$$
\begin{aligned}
& \left\|v\left(T_{1}, \theta_{-t} \omega, v_{0}\left(\theta_{-t} \omega\right)\right)\right\|^{2} \\
& \quad \leq e^{-\gamma T_{1}}\left\|v_{0}\left(\theta_{-t} \omega\right)\right\|^{2}+\int_{0}^{T_{1}} e^{\gamma\left(s-T_{1}\right)} h\left(\theta_{s-t} \omega\right) \mathrm{d} s .
\end{aligned}
$$

With (18) and (26) in mind, by multiplying $e^{\gamma\left(T_{1}-t\right)}$ at both sides of the above equation, one can easily get

$$
\begin{aligned}
& e^{\gamma\left(T_{1}-t\right)}\left\|v\left(T_{1}, \theta_{-t} \omega, v_{0}\left(\theta_{-t} \omega\right)\right)\right\|^{2} \\
& \leq e^{-\gamma t}\left\|v_{0}\left(\theta_{-t} \omega\right)\right\|^{2}+\int_{0}^{T_{1}} e^{\gamma(s-t)} h\left(\theta_{s-t} \omega\right) \mathrm{d} s \\
& \leq e^{-\gamma t}\left\|v_{0}\left(\theta_{-t} \omega\right)\right\|^{2}+\int_{-t}^{T_{1}-t} e^{\gamma \tau} h\left(\theta_{\tau} \omega\right) \mathrm{d} \tau \\
& \leq e^{-\gamma t}\left\|v_{0}\left(\theta_{-t} \omega\right)\right\|^{2}+c \cdot r(\omega) \int_{-t}^{T_{1}-t} e^{(\gamma / 2) \tau} \mathrm{d} \tau \\
& \leq e^{-\gamma t}\left\|v_{0}\left(\theta_{-t} \omega\right)\right\|^{2}+\frac{c}{\gamma} \cdot r(\omega) e^{(\gamma / 2)\left(T_{1}-t\right)} .
\end{aligned}
$$


From (25) to (26),

$$
\begin{aligned}
\frac{d}{d t}\|v\|^{2} & +\lambda\|\nabla v\|^{2}+\gamma\|v\|^{2}+\kappa\|u\|_{4}^{4} \\
\leq & \frac{d}{d t}\|v\|^{2}+\lambda\|\nabla v\|^{2}+2 \gamma\|v\|^{2} \\
& +\kappa\|u\|_{4}^{4} \leq h\left(\theta_{t} \omega\right) .
\end{aligned}
$$

Multiply (37) by $e^{\gamma(s-t)}$ and then integrate from $T_{1}$ to $t$; we then obtain

$$
\begin{aligned}
& \left\|v\left(t, \omega, v_{0}(\omega)\right)\right\|^{2}+\lambda \cdot \int_{T_{1}}^{t} e^{\gamma(s-t)}\left\|\nabla v\left(s, \omega, v_{0}(\omega)\right)\right\|^{2} \mathrm{~d} s \\
& \quad+\kappa \cdot \int_{T_{1}}^{t} e^{\gamma(s-t)}\left\|u\left(s, \omega, u_{0}(\omega)\right)\right\|_{4}^{4} \mathrm{~d} s \\
& \leq e^{\gamma\left(T_{1}-t\right)}\left\|v\left(T_{1}, \omega, v_{0}(\omega)\right)\right\|^{2} \\
& \quad+\int_{T_{1}}^{t} e^{\gamma(s-t)} h\left(\theta_{s} \omega\right) \mathrm{d} s .
\end{aligned}
$$

Keep the last two terms on the left-hand side of (38), and replace $\omega$ by $\theta_{-t} \omega$; we then have

$$
\begin{aligned}
& \lambda \cdot \int_{T_{1}}^{t} e^{\gamma(s-t)}\left\|\nabla v\left(s, \theta_{-t} \omega, v_{0}\left(\theta_{-t} \omega\right)\right)\right\|^{2} \mathrm{~d} s \\
& \quad+\kappa \cdot \int_{T_{1}}^{t} e^{\gamma(s-t)}\left\|u\left(s, \theta_{-t} \omega, u_{0}\left(\theta_{-t} \omega\right)\right)\right\|_{4}^{4} \mathrm{~d} s \\
& \leq e^{\gamma\left(T_{1}-t\right)}\left\|v\left(T_{1}, \theta_{-t} \omega, v_{0}\left(\theta_{-t} \omega\right)\right)\right\|^{2} \\
& \quad+\int_{T_{1}}^{t} e^{\gamma(s-t)} h\left(\theta_{s-t} \omega\right) \mathrm{d} s \\
& \leq e^{\gamma\left(T_{1}-t\right)}\left\|v\left(T_{1}, \theta_{-t} \omega, v_{0}\left(\theta_{-t} \omega\right)\right)\right\|^{2} \\
& \quad+\int_{T_{1}-t}^{0} e^{\gamma \tau} h\left(\theta_{\tau} \omega\right) \mathrm{d} \tau .
\end{aligned}
$$

However the second term on the right-hand side can be bounded by

$$
c \cdot r(\omega) \int_{T_{1}-t}^{0} e^{(\gamma / 2) \tau} \mathrm{d} \tau \leq \frac{c}{\gamma} \cdot r(\omega),
$$

due to (18) and (26). Together with (36), there is

$$
\begin{aligned}
& \lambda \cdot \int_{T_{1}}^{t} e^{\gamma(s-t)}\left\|\nabla v\left(s, \theta_{-t} \omega, v_{0}\left(\theta_{-t} \omega\right)\right)\right\|^{2} \mathrm{~d} s \\
& \quad+\kappa \cdot \int_{T_{1}}^{t} e^{\gamma(s-t)}\left\|u\left(s, \theta_{-t} \omega, u_{0}\left(\theta_{-t} \omega\right)\right)\right\|_{4}^{4} \mathrm{~d} s \\
& \leq e^{-\gamma t}\left\|v_{0}\left(\theta_{-t} \omega\right)\right\|^{2}+\frac{c}{\gamma} \cdot r(\omega) e^{(\gamma / 2)\left(T_{1}-t\right)}+\frac{c}{\gamma} \cdot r(\omega) \\
& \leq e^{-\gamma t}\left\|v_{0}\left(\theta_{-t} \omega\right)\right\|^{2}+\frac{2 c}{\gamma} \cdot r(\omega) \quad \forall t \geq T_{1} .
\end{aligned}
$$

The proof is completed.
Corollary 13. Let $B=\{B(\omega)\}_{\omega \in \Omega} \in \mathscr{D}$ and $u_{0}(\omega) \in B(\omega)$, and then, for P-a.e. $\omega \in \Omega$, there exists $t_{B}(\omega)>0$ such that the solutions $u\left(t, \omega, u_{0}(\omega)\right)$ of (1)-(2) and $v\left(t, \omega, v_{0}(\omega)\right)$ of (20), with $v_{0}(\omega)=u_{0}(\omega)-z(\omega)$, satisfy the following uniform estimates, for all $t \geq t_{B}(\omega)$ :

$$
\begin{aligned}
& \int_{t}^{t+1}\left\|u\left(s, \theta_{-t-1} \omega, u_{0}\left(\theta_{-t-1} \omega\right)\right)\right\|_{4}^{4} d s \leq \frac{4 c}{\gamma} \cdot e^{\gamma} \cdot r(\omega), \\
& \int_{t}^{t+1}\left\|\nabla v\left(s, \theta_{-t-1} \omega, v_{0}\left(\theta_{-t-1} \omega\right)\right)\right\|^{2} d s \leq \frac{4 c}{\gamma} \cdot e^{\gamma} \cdot r(\omega) .
\end{aligned}
$$

Proof. Replace $t$ by $(t+1)$ and then replace $T_{1}$ by $t$ in (34); we then deduce

$$
\begin{aligned}
& e^{-\gamma} \int_{t}^{t+1}\left\|u\left(s, \theta_{-t-1} \omega, u_{0}\left(\theta_{-t-1} \omega\right)\right)\right\|_{4}^{4} \mathrm{~d} s \\
& \leq \int_{t}^{t+1} e^{\gamma(s-t-1)}\left\|u\left(s, \theta_{-t-1} \omega, u_{0}\left(\theta_{-t-1} \omega\right)\right)\right\|_{4}^{4} \mathrm{~d} s \\
& \leq \frac{1}{\kappa} e^{-\gamma(t+1)}\left\|v_{0}\left(\theta_{-t-1} \omega\right)\right\|^{2}+\frac{2 c}{\gamma \kappa} \cdot r(\omega) \\
& \leq \frac{2}{\kappa} e^{-\gamma(t+1)}\left(\left\|u_{0}\left(\theta_{-t-1} \omega\right)\right\|^{2}+\left\|z\left(\theta_{-t-1} \omega\right)\right\|^{2}\right) \\
& \quad+\frac{2 c}{\gamma} \cdot r(\omega) .
\end{aligned}
$$

As both random variables $u_{0}(\omega) \in B(\omega)$ and $z(\omega)$ are tempered, there exists $t_{B}(\omega)>0$, such that, for all $t \geq t_{B}(\omega)$,

$$
\frac{2}{\kappa} e^{-\gamma(t+1)}\left(\left\|u_{0}\left(\theta_{-t-1} \omega\right)\right\|^{2}+\left\|z\left(\theta_{-t-1} \omega\right)\right\|^{2}\right) \leq \frac{2 c}{\gamma} \cdot r(\omega),
$$

which, together with (43), claims that, for all $t \geq t_{B}(\omega)$,

$$
\int_{t}^{t+1}\left\|u\left(s, \theta_{-t-1} \omega, u_{0}\left(\theta_{-t-1} \omega\right)\right)\right\|_{4}^{4} \mathrm{~d} s \leq \frac{4 c}{\gamma} \cdot e^{\gamma} \cdot r(\omega)
$$

With the same procedure as the above, we can also verify that, for all $t \geq t_{B}(\omega)$,

$$
\int_{t}^{t+1}\left\|\nabla v\left(s, \theta_{-t-1} \omega, v_{0}\left(\theta_{-t-1} \omega\right)\right)\right\|^{2} \mathrm{~d} s \leq \frac{4 c}{\gamma} \cdot e^{\gamma} \cdot r(\omega) .
$$

The proof is completed.

Corollary 14. Let $B=\{B(\omega)\}_{\omega \in \Omega} \in \mathscr{D}$ and $u_{0}(\omega) \in B(\omega)$, and then, for $P$-a.e. $\omega \in \Omega$, there exists $t_{B}(\omega)>0$ such that the solution $u\left(t, \omega, u_{0}(\omega)\right)$ of (1)-(2) satisfies

$$
\begin{gathered}
\int_{t}^{t+1}\left\|\nabla u\left(s, \theta_{-t-1} \omega, u_{0}\left(\theta_{-t-1} \omega\right)\right)\right\|^{2} d s \\
\leq \frac{4 c}{\gamma} \cdot e^{\gamma} \cdot r(\omega), \quad \forall t \geq t_{B}(\omega) .
\end{gathered}
$$


Proof. Let $t_{B}(\omega)>0$ just be the one in Corollary 13, and take $t \geq t_{B}(\omega)$ and $s \in(t, t+1)$. Note that by (21) one has

$$
\begin{aligned}
\| \nabla u & \left(s, \theta_{-t-1} \omega, u_{0}\left(\theta_{-t-1} \omega\right)\right) \|^{2} \\
= & \left\|\nabla v\left(s, \theta_{-t-1} \omega, v_{0}\left(\theta_{-t-1} \omega\right)\right)+\nabla z\left(\theta_{s-t-1} \omega\right)\right\|^{2} \\
\leq & 2\left\|\nabla v\left(s, \theta_{-t-1} \omega, v_{0}\left(\theta_{-t-1} \omega\right)\right)\right\|^{2} \\
& +2\left\|\nabla z\left(\theta_{s-t-1} \omega\right)\right\|^{2} .
\end{aligned}
$$

Owing to (18), one has

$$
\begin{aligned}
2\left\|\nabla z\left(\theta_{s-t-1} \omega\right)\right\|^{2} & \leq c \cdot \sum_{j=1}^{m}\left|z_{j}\left(\theta_{s-t-1} \omega\right)\right|^{2} \\
& \leq c e^{(\gamma / 2)(t+1-s)} r(\omega) \leq c e^{\gamma / 2} r(\omega) .
\end{aligned}
$$

Together with Corollary 13, we derive

$$
\begin{aligned}
\int_{t}^{t+1} & \left\|\nabla u\left(s, \theta_{-t-1} \omega, u_{0}\left(\theta_{-t-1} \omega\right)\right)\right\|^{2} \mathrm{~d} s \\
\leq & 2 \int_{t}^{t+1}\left\|\nabla v\left(s, \theta_{-t-1} \omega, v_{0}\left(\theta_{-t-1} \omega\right)\right)\right\|^{2} \mathrm{~d} s \\
& +2 \int_{t}^{t+1}\left\|\nabla z\left(\theta_{s-t-1} \omega\right)\right\|^{2} \mathrm{~d} s \\
\leq & \frac{4 c}{\gamma} \cdot e^{\gamma} \cdot r(\omega)+c e^{\gamma / 2} r(\omega) \\
\leq & \frac{4 c}{\gamma} \cdot e^{\gamma} \cdot r(\omega),
\end{aligned}
$$

by integrating (48) with respect to $s$ over $(t, t+1)$.

The proof is completed.

Lemma 15. Suppose $\sqrt{3} \kappa \geq|\beta|$, and let $B=\{B(\omega)\}_{\omega \in \Omega} \in \mathscr{D}$ and $u_{0}(\omega) \in B(\omega)$; then, for $P$-a.e. $\omega \in \Omega$, there exists $t_{B}(\omega)>$ 0 such that, for all $t \geq t_{B}(\omega)$,

$$
\left\|\nabla u\left(t, \theta_{-t} \omega, u_{0}\left(\theta_{-t} \omega\right)\right)\right\|^{2} \leq \frac{4 c}{\gamma} \cdot e^{\gamma} \cdot r(\omega) .
$$

Proof. By multiplying (20) by $\Delta \bar{v}$, integrating over $\mathbb{R}^{n}$, and then taking the real part, we get

$$
\begin{gathered}
\frac{1}{2} \cdot \frac{d}{d t}\|\nabla v\|^{2}+\lambda\|\Delta v\|^{2}+\gamma\|\nabla v\|^{2} \\
=\operatorname{Re}\left((\kappa+i \beta)\left(|v+z|^{2}(v+z), \Delta \bar{v}\right)\right) \\
-\operatorname{Re}\left((\lambda+i \mu)\left(\Delta z\left(\theta_{t} \omega\right), \Delta \bar{v}\right)\right) .
\end{gathered}
$$

Since

$$
\left(|v+z|^{2}(v+z), \Delta \bar{v}\right)=\left(|u|^{2} u, \Delta \bar{u}\right)-\left(|u|^{2} u, \Delta \bar{z}\left(\theta_{t} \omega\right)\right),
$$

while

$$
\left(|u|^{2} u, \Delta \bar{u}\right)=-\int_{\mathbb{R}^{n}}\left(|u|^{2}|\nabla u|^{2}+u \nabla \bar{u} \nabla|u|^{2}\right) \mathrm{d} x,
$$

we have

$$
\begin{aligned}
\operatorname{Re}\left((\kappa+i \beta)\left(|u|^{2} u, \Delta \bar{u}\right)\right) \\
=-\kappa \int_{\mathbb{R}^{n}}|u|^{2}|\nabla u|^{2} \mathrm{~d} x-\kappa \int_{\mathbb{R}^{n}} \operatorname{Re}\left(u \nabla \bar{u} \nabla|u|^{2}\right) \mathrm{d} x \\
\quad+\beta \int_{\mathbb{R}^{n}} \operatorname{Im}\left(u \nabla \bar{u} \nabla|u|^{2}\right) \mathrm{d} x \\
=-\kappa \int_{\mathbb{R}^{n}}|u|^{2}|\nabla u|^{2} \mathrm{~d} x-\frac{\kappa}{2} \int_{\mathbb{R}^{n}}\left(\nabla|u|^{2}\right)^{2} \mathrm{~d} x \\
\quad-\frac{\beta}{2} \int_{\mathbb{R}^{n}} i(u \nabla \bar{u}-\bar{u} \nabla u) \nabla|u|^{2} \mathrm{~d} x \\
=-\frac{1}{4} \int_{\mathbb{R}^{n}}\left(3 \kappa\left(\nabla|u|^{2}\right)^{2}+2 \beta i(u \nabla \bar{u}-\bar{u} \nabla u) \nabla|u|^{2}\right. \\
\left.\quad+\kappa|u \nabla \bar{u}-\bar{u} \nabla u|^{2}\right) \mathrm{d} x
\end{aligned}
$$

$\leq 0$,

provided that $\sqrt{3} \kappa \geq|\beta|$.

Therefore, for the first term at the right-hand side of (52), we have

$$
\begin{aligned}
\operatorname{Re} & \left((\kappa+i \beta)\left(|v+z|^{2}(v+z), \Delta \bar{v}\right)\right) \\
= & \operatorname{Re}\left((\kappa+i \beta)\left(|u|^{2} u, \Delta \bar{u}\right)\right) \\
& -\operatorname{Re}\left((\kappa+i \beta)\left(|u|^{2} u, \Delta \bar{z}\left(\theta_{t} \omega\right)\right)\right) \\
\leq & -\operatorname{Re}\left((\kappa+i \beta)\left(|u|^{2} u, \Delta \bar{z}\left(\theta_{t} \omega\right)\right)\right) \\
\leq & |\kappa+i \beta| \cdot \int_{\mathbb{R}^{n}}|u|^{3} \cdot\left|\Delta z\left(\theta_{t} \omega\right)\right| \mathrm{d} x \\
\leq & \frac{3}{4}\|u\|_{4}^{4}+\frac{1}{4}\left(\kappa^{2}+\beta^{2}\right)^{2} \cdot\left\|\Delta z\left(\theta_{t} \omega\right)\right\|_{4}^{4} .
\end{aligned}
$$

On the other hand, the second term at the right-hand side of (52) can be bounded by

$$
\begin{aligned}
\mid \lambda & +i \mu\left|\cdot \int_{\mathbb{R}^{n}}\right| \Delta z\left(\theta_{t} \omega\right)|\cdot| \Delta v \mid \mathrm{d} x \\
& \leq \lambda\|\Delta v\|^{2}+\frac{\lambda^{2}+\mu^{2}}{4 \lambda}\left\|\Delta z\left(\theta_{t} \omega\right)\right\|^{2} .
\end{aligned}
$$

By (52), (56)-(57), we can see that

$$
\begin{aligned}
\frac{d}{d t}\|\nabla v\|^{2} & +2 \gamma\|\nabla v\|^{2} \\
\leq & \frac{3}{2}\|u\|_{4}^{4}+\frac{1}{2}\left(\kappa^{2}+\beta^{2}\right)^{2} \\
& \cdot\left\|\Delta z\left(\theta_{t} \omega\right)\right\|_{4}^{4}+\frac{\lambda^{2}+\mu^{2}}{2 \lambda}\left\|\Delta z\left(\theta_{t} \omega\right)\right\|^{2}
\end{aligned}
$$

That is,

$$
\frac{d}{d t}\|\nabla v\|^{2} \leq \frac{3}{2}\|u\|_{4}^{4}+g\left(\theta_{t} \omega\right)
$$


where

$$
g\left(\theta_{t} \omega\right) \triangleq \frac{1}{2}\left(\kappa^{2}+\beta^{2}\right)^{2} \cdot\left\|\Delta z\left(\theta_{t} \omega\right)\right\|_{4}^{4}+\frac{\lambda^{2}+\mu^{2}}{2 \lambda}\left\|\Delta z\left(\theta_{t} \omega\right)\right\|^{2} .
$$

Since $z\left(\theta_{t} \omega\right)=\sum_{j=1}^{m} \varphi_{j} z_{j}\left(\theta_{t} \omega_{j}\right)$, where $\varphi_{j} \in H^{2}\left(\mathbb{R}^{n}\right) \cap$ $W^{2,4}\left(\mathbb{R}^{n}\right)$, there exists a constant $c>0$ such that

$$
\begin{aligned}
g\left(\theta_{t} \omega\right) & \leq c \cdot \sum_{j=1}^{m}\left(\left|z_{j}\left(\theta_{t} \omega_{j}\right)\right|^{2}+\left|z_{j}\left(\theta_{t} \omega_{j}\right)\right|^{4}\right) \\
& \leq c \cdot e^{(\gamma / 2)|t|} r(\omega), \quad \forall t \in \mathbb{R} .
\end{aligned}
$$

Let $t>t_{B}(\omega), s \in(t, t+1)$, where $t_{B}(\omega)$ is the positive time taken in Corollary 13. By integrating (59) from $s$ to $t+1$, we obtain

$$
\begin{aligned}
\left\|\nabla v\left(t+1, \omega, v_{0}(\omega)\right)\right\|^{2} \leq & \left\|\nabla v\left(s, \omega, v_{0}(\omega)\right)\right\|^{2} \\
& +\frac{3}{2} \int_{s}^{t+1}\left\|u\left(\tau, \omega, u_{0}(\omega)\right)\right\|_{4}^{4} \mathrm{~d} \tau \\
& +\int_{s}^{t+1} g\left(\theta_{\tau} \omega\right) \mathrm{d} \tau .
\end{aligned}
$$

Integrate the above equation with respect to $s$ over $(t, t+1)$ to have

$$
\begin{aligned}
\left\|\nabla v\left(t+1, \omega, v_{0}(\omega)\right)\right\|^{2} \leq & \int_{t}^{t+1}\left\|\nabla v\left(s, \omega, v_{0}(\omega)\right)\right\|^{2} \mathrm{~d} s \\
& +\frac{3}{2} \int_{t}^{t+1}\left\|u\left(\tau, \omega, u_{0}(\omega)\right)\right\|_{4}^{4} \mathrm{~d} \tau \\
& +\int_{t}^{t+1} g\left(\theta_{\tau} \omega\right) \mathrm{d} \tau .
\end{aligned}
$$

By replacing $\omega$ by $\theta_{-t-1} \omega$, we derive

$$
\begin{aligned}
& \left\|\nabla v\left(t+1, \theta_{-t-1} \omega, v_{0}\left(\theta_{-t-1} \omega\right)\right)\right\|^{2} \\
& \leq \int_{t}^{t+1}\left\|\nabla v\left(s, \theta_{-t-1} \omega, v_{0}\left(\theta_{-t-1} \omega\right)\right)\right\|^{2} \mathrm{~d} s \\
& \quad+\frac{3}{2} \int_{t}^{t+1}\left\|u\left(\tau, \theta_{-t-1} \omega, u_{0}\left(\theta_{-t-1} \omega\right)\right)\right\|_{4}^{4} \mathrm{~d} \tau \\
& \quad+\int_{t}^{t+1} g\left(\theta_{\tau-t-1} \omega\right) \mathrm{d} \tau .
\end{aligned}
$$

Thanks to Corollary 13, it follows from (61) and (64) that, for all $t>t_{B}(\omega)$,

$$
\begin{aligned}
& \left\|\nabla v\left(t+1, \theta_{-t-1} \omega, v_{0}\left(\theta_{-t-1} \omega\right)\right)\right\|^{2} \\
& \quad \leq \frac{4 c}{\gamma} \cdot e^{\gamma} \cdot r(\omega)+\frac{3}{2} \cdot \frac{4 c}{\gamma} \cdot e^{\gamma} \cdot r(\omega)+\int_{-1}^{0} g\left(\theta_{\tau} \omega\right) \mathrm{d} \tau
\end{aligned}
$$

$$
\begin{aligned}
& \leq \frac{4 c}{\gamma} \cdot e^{\gamma} \cdot r(\omega)+c \cdot r(\omega) \int_{-1}^{0} e^{-(\gamma / 2) \tau} \mathrm{d} \tau \\
& \leq \frac{4 c}{\gamma} \cdot e^{\gamma} \cdot r(\omega) .
\end{aligned}
$$

Then, together with (16), we obtain that, for all $t>t_{B}(\omega)$,

$$
\begin{aligned}
&\left\|\nabla u\left(t+1, \theta_{-t-1} \omega, u_{0}\left(\theta_{-t-1} \omega\right)\right)\right\|^{2} \\
&=\left\|\nabla v\left(t+1, \theta_{-t-1} \omega, v_{0}\left(\theta_{-t-1} \omega\right)\right)+\nabla z(\omega)\right\|^{2} \\
& \leq 2\left\|\nabla v\left(t+1, \theta_{-t-1} \omega, v_{0}\left(\theta_{-t-1} \omega\right)\right)\right\|^{2} \\
&+2\|\nabla z(\omega)\|^{2} \\
& \leq \frac{4 c}{\gamma} \cdot e^{\gamma} \cdot r(\omega) .
\end{aligned}
$$

The proof is completed.

Lemma 16. Suppose $\sqrt{3} \kappa \geq|\beta|$, and let $B=\{B(\omega)\}_{\omega \in \Omega} \in \mathscr{D}$ and $u_{0}(\omega) \in B(\omega)$; then, for every $\epsilon>0$ and $P$-a.e. $\omega \in \Omega$, there exist $T^{*}=T(B, \omega, \epsilon)>0$ and $R^{*}=R^{*}(\omega, \epsilon)$ such that the solution $v\left(t, \omega, v_{0}(\omega)\right)$ of $(20)$ with $v_{0}(\omega)=u_{0}(\omega)-z(\omega)$ satisfies, for all $t \geq T^{*}$,

$$
\int_{|x| \geq R^{*}}\left|v\left(t, \theta_{-t} \omega, v_{0}\left(\theta_{-t} \omega\right)\right)(x)\right|^{2} d x \leq \epsilon .
$$

Proof. Let $\rho$ be a smooth function defined on $\mathbb{R}^{+}$such that $0 \leq \rho(s) \leq 1$ for all $s \in \mathbb{R}^{+}$, and

$$
\rho(s)= \begin{cases}0 & \text { for } 0 \leq s \leq 1, \\ 1 & \text { for } s \geq 2\end{cases}
$$

Then there exists a constant $c>0$ such that $\left|\rho^{\prime}(s)\right| \leq c$, for all $s \in \mathbb{R}^{+}$. Multiply (20) by $\rho\left(|x|^{2} / l^{2}\right) \bar{v}$, integrate over $\mathbb{R}^{n}$, and then take the real part to get

$$
\begin{aligned}
& \frac{1}{2} \frac{d}{d t} \int_{\mathbb{R}^{n}} \rho\left(\frac{\left|x^{2}\right|}{l^{2}}\right)|v|^{2} \mathrm{~d} x \\
& =\operatorname{Re}\left((\lambda+i \mu) \int_{\mathbb{R}^{n}} \rho\left(\frac{\left|x^{2}\right|}{l^{2}}\right) \Delta v \bar{v} \mathrm{~d} x\right) \\
& \quad-\operatorname{Re}\left((\kappa+i \beta) \int_{\mathbb{R}^{n}} \rho\left(\frac{\left|x^{2}\right|}{l^{2}}\right)|v+z|^{2}(v+z) \bar{v} \mathrm{~d} x\right) \\
& \quad-\gamma \int_{\mathbb{R}^{n}} \rho\left(\frac{\left|x^{2}\right|}{l^{2}}\right)|v|^{2} \mathrm{~d} x \\
& \quad+\operatorname{Re}\left((\lambda+i \mu) \int_{\mathbb{R}^{n}} \rho\left(\frac{\left|x^{2}\right|}{l^{2}}\right) \Delta z \bar{v} \mathrm{~d} x\right) .
\end{aligned}
$$


We now concentrate to estimate the terms in (69). Firstly,

$$
\begin{aligned}
\int_{\mathbb{R}^{n}} \rho\left(\frac{\left|x^{2}\right|}{l^{2}}\right) \Delta v \bar{v} \mathrm{~d} x \\
=-\int_{\mathbb{R}^{n}}|\nabla v|^{2} \rho\left(\frac{\left|x^{2}\right|}{l^{2}}\right) \mathrm{d} x-\int_{\mathbb{R}^{n}} \bar{v} \rho^{\prime}\left(\frac{\left|x^{2}\right|}{l^{2}}\right) \frac{2 x}{l^{2}} \nabla v \mathrm{~d} x \\
=-\int_{\mathbb{R}^{n}}|\nabla v|^{2} \rho\left(\frac{\left|x^{2}\right|}{l^{2}}\right) \mathrm{d} x \\
\quad-\int_{l \leq|x| \leq \sqrt{2} l} \bar{v} \rho^{\prime}\left(\frac{\left|x^{2}\right|}{l^{2}}\right) \frac{2 x}{l^{2}} \nabla v \mathrm{~d} x .
\end{aligned}
$$

Since

$$
\begin{aligned}
& \left|\int_{l \leq|x| \leq \sqrt{2} l} \bar{v} \rho^{\prime}\left(\frac{\left|x^{2}\right|}{l^{2}}\right) \frac{2 x}{l^{2}} \nabla v \mathrm{~d} x\right| \\
& \quad \leq \frac{2 \sqrt{2}}{l} \int_{l \leq|x| \leq \sqrt{2} l}|v| \cdot\left|\rho^{\prime}\left(\frac{\left|x^{2}\right|}{l^{2}}\right)\right| \cdot|\nabla v| \mathrm{d} x \\
& \quad \leq \frac{c}{l} \int_{\mathbb{R}^{n}}|v| \cdot|\nabla v| \mathrm{d} x \\
& \quad \leq \frac{c}{l}\left(\|v\|^{2}+\|\nabla v\|^{2}\right),
\end{aligned}
$$

then we find that

$$
\begin{gathered}
\operatorname{Re}\left((\lambda+i \mu) \int_{\mathbb{R}^{n}} \rho\left(\frac{\left|x^{2}\right|}{l^{2}}\right) \Delta v \bar{v} \mathrm{~d} x\right) \\
\leq-\lambda \cdot \int_{\mathbb{R}^{n}}|\nabla v|^{2} \rho\left(\frac{\left|x^{2}\right|}{l^{2}}\right) \mathrm{d} x \\
+\frac{c \cdot|\lambda+i \mu|}{l}\left(\|v\|^{2}+\|\nabla v\|^{2}\right) \\
\leq-\lambda \cdot \int_{\mathbb{R}^{n}}|\nabla v|^{2} \rho\left(\frac{\left|x^{2}\right|}{l^{2}}\right) \mathrm{d} x \\
+\frac{c}{l}\left(\|v\|^{2}+\|\nabla v\|^{2}\right) .
\end{gathered}
$$

Secondly,

$$
\begin{aligned}
\int_{\mathbb{R}^{n}} \rho\left(\frac{\left|x^{2}\right|}{l^{2}}\right)|v+z|^{2}(v+z) \bar{v} \mathrm{~d} x \\
=\int_{\mathbb{R}^{n}} \rho\left(\frac{\left|x^{2}\right|}{l^{2}}\right)|u|^{4} \mathrm{~d} x \\
\quad-\int_{\mathbb{R}^{n}} \rho\left(\frac{\left|x^{2}\right|}{l^{2}}\right)|u|^{2} \cdot u \cdot \bar{z}\left(\theta_{t} \omega\right) \mathrm{d} x
\end{aligned}
$$

Due to

$$
\begin{aligned}
& \left.\left|\int_{\mathbb{R}^{n}} \rho\left(\frac{\left|x^{2}\right|}{l^{2}}\right)\right| u\right|^{2} \cdot u \cdot \bar{z}\left(\theta_{t} \omega\right) \mathrm{d} x \mid \\
& \quad \leq \int_{\mathbb{R}^{n}} \rho\left(\frac{\left|x^{2}\right|}{l^{2}}\right)|u|^{3} \cdot\left|z\left(\theta_{t} \omega\right)\right| \mathrm{d} x
\end{aligned}
$$

$$
\begin{aligned}
& \leq \frac{\kappa}{2|\kappa+i \beta|} \int_{\mathbb{R}^{n}} \rho\left(\frac{\left|x^{2}\right|}{l^{2}}\right)|u|^{4} \mathrm{~d} x \\
& \quad+\frac{c}{|\kappa+i \beta|} \int_{\mathbb{R}^{n}} \rho\left(\frac{\left|x^{2}\right|}{l^{2}}\right)\left|z\left(\theta_{t} \omega\right)\right|^{4} \mathrm{~d} x,
\end{aligned}
$$

we have

$$
\begin{aligned}
- & \operatorname{Re}\left((\kappa+i \beta) \int_{\mathbb{R}^{n}} \rho\left(\frac{\left|x^{2}\right|}{l^{2}}\right)|v+z|^{2}(v+z) \bar{v} \mathrm{~d} x\right) \\
= & -\kappa \cdot \int_{\mathbb{R}^{n}} \rho\left(\frac{\left|x^{2}\right|}{l^{2}}\right)|u|^{4} \mathrm{~d} x \\
& +\operatorname{Re}\left((\kappa+i \beta) \int_{\mathbb{R}^{n}} \rho\left(\frac{\left|x^{2}\right|}{l^{2}}\right)|u|^{2} \cdot u \cdot \bar{z}\left(\theta_{t} \omega\right) \mathrm{d} x\right) \\
\leq & -\kappa \cdot \int_{\mathbb{R}^{n}} \rho\left(\frac{\left|x^{2}\right|}{l^{2}}\right)|u|^{4} \mathrm{~d} x+\frac{\kappa}{2} \int_{\mathbb{R}^{n}} \rho\left(\frac{\left|x^{2}\right|}{l^{2}}\right)|u|^{4} \mathrm{~d} x \\
& +c \cdot \int_{\mathbb{R}^{n}} \rho\left(\frac{\left|x^{2}\right|}{l^{2}}\right)\left|z\left(\theta_{t} \omega\right)\right|^{4} \mathrm{~d} x \\
\leq & -\frac{\kappa}{2} \int_{\mathbb{R}^{n}} \rho\left(\frac{\left|x^{2}\right|}{l^{2}}\right)|u|^{4} \mathrm{~d} x \\
& +c \cdot \int_{\mathbb{R}^{n}} \rho\left(\frac{\left|x^{2}\right|}{l^{2}}\right)\left|z\left(\theta_{t} \omega\right)\right|^{4} \mathrm{~d} x .
\end{aligned}
$$

Thirdly,

$$
\begin{aligned}
& \operatorname{Re}\left((\lambda+i \mu) \int_{\mathbb{R}^{n}} \rho\left(\frac{\left|x^{2}\right|}{l^{2}}\right) \Delta z \bar{v} \mathrm{~d} x\right) \\
& \leq \frac{\gamma}{2} \int_{\mathbb{R}^{n}} \rho\left(\frac{\left|x^{2}\right|}{l^{2}}\right)|v|^{2} \mathrm{~d} x \\
& +\frac{\lambda^{2}+\mu^{2}}{2 \gamma} \int_{\mathbb{R}^{n}} \rho\left(\frac{\left|x^{2}\right|}{l^{2}}\right)|\Delta z|^{2} \mathrm{~d} x .
\end{aligned}
$$

Finally, from (69) to (76),

$$
\begin{aligned}
& \frac{1}{2} \frac{d}{d t} \int_{\mathbb{R}^{n}} \rho\left(\frac{\left|x^{2}\right|}{l^{2}}\right)|v|^{2} \mathrm{~d} x+\frac{\gamma}{2} \int_{\mathbb{R}^{n}} \rho\left(\frac{\left|x^{2}\right|}{l^{2}}\right)|v|^{2} \mathrm{~d} x \\
& \quad+\frac{\kappa}{2} \int_{\mathbb{R}^{n}} \rho\left(\frac{\left|x^{2}\right|}{l^{2}}\right)|u|^{4} \mathrm{~d} x+\lambda \cdot \int_{\mathbb{R}^{n}}|\nabla v|^{2} \rho\left(\frac{\left|x^{2}\right|}{l^{2}}\right) \mathrm{d} x \\
& \leq \frac{c}{l}\left(\|v\|^{2}+\|\nabla v\|^{2}\right)+c \cdot \int_{\mathbb{R}^{n}} \rho\left(\frac{\left|x^{2}\right|}{l^{2}}\right)\left|z\left(\theta_{t} \omega\right)\right|^{4} \mathrm{~d} x \\
& +\frac{\lambda^{2}+\mu^{2}}{2 \gamma} \int_{\mathbb{R}^{n}} \rho\left(\frac{\left|x^{2}\right|}{l^{2}}\right)|\Delta z|^{2} \mathrm{~d} x,
\end{aligned}
$$


which implies

$$
\begin{aligned}
& \frac{d}{d t} \int_{\mathbb{R}^{n}} \rho\left(\frac{\left|x^{2}\right|}{l^{2}}\right)|v|^{2} \mathrm{~d} x+\gamma \int_{\mathbb{R}^{n}} \rho\left(\frac{\left|x^{2}\right|}{l^{2}}\right)|v|^{2} \mathrm{~d} x \\
& \leq \frac{c}{l}\left(\|v\|^{2}+\|\nabla v\|^{2}\right)+c \cdot \int_{\mathbb{R}^{n}} \rho\left(\frac{\left|x^{2}\right|}{l^{2}}\right)\left|z\left(\theta_{t} \omega\right)\right|^{4} \mathrm{~d} x \\
& +\frac{\lambda^{2}+\mu^{2}}{\gamma} \int_{\mathbb{R}^{n}} \rho\left(\frac{\left|x^{2}\right|}{l^{2}}\right)|\Delta z|^{2} \mathrm{~d} x .
\end{aligned}
$$

Proposition 11 together with Lemma 15 shows that there is $T_{1}=t_{B}(\omega)$ such that, for all $t \geq T_{1}$,

$$
\left\|v\left(t, \theta_{-t} \omega, v_{0}\left(\theta_{-t} \omega\right)\right)\right\|_{H^{1}\left(\mathbb{R}^{n}\right)}^{2} \leq \frac{4 c}{\gamma} \cdot e^{\gamma} \cdot r(\omega) .
$$

Now, multiply (78) with $e^{\gamma(s-t)}$, and then integrate over $\left(T_{1}, t\right)$ with respect to $s$ so that, for all $t \geq T_{1}$,

$$
\begin{aligned}
& \int_{\mathbb{R}^{n}} \rho\left(\frac{\left|x^{2}\right|}{l^{2}}\right)\left|v\left(t, \omega, v_{0}(\omega)\right)\right|^{2} \mathrm{~d} x \\
& \leq e^{\gamma\left(T_{1}-t\right)} \int_{\mathbb{R}^{n}} \rho\left(\frac{\left|x^{2}\right|}{l^{2}}\right)\left|v\left(T_{1}, \omega, v_{0}(\omega)\right)\right|^{2} \mathrm{~d} x \\
& +\frac{c}{l} \int_{T_{1}}^{t} e^{\gamma(s-t)}\left(\left\|v\left(s, \omega, v_{0}(\omega)\right)\right\|^{2}\right. \\
& +c \cdot \int_{T_{1}}^{t} e^{\gamma(s-t)} \int_{\mathbb{R}^{n}} \rho\left(\frac{\left|x^{2}\right|}{l^{2}}\right)\left|z\left(\theta_{s} \omega\right)\right|^{4} \mathrm{~d} x \mathrm{~d} s \\
& +\frac{\lambda^{2}+\mu^{2}}{\gamma} \int_{T_{1}}^{t} e^{\gamma(s-t)} \int_{\mathbb{R}^{n}} \rho\left(\frac{\left|x^{2}\right|}{l^{2}}\right)\left|\Delta z\left(\theta_{s} \omega\right)\right|^{2} \mathrm{~d} x \mathrm{~d} s
\end{aligned}
$$

By replacing $\omega$ by $\theta_{-t} \omega$ in (80), we obtain that, for all $t \geq T_{1}$,

$$
\begin{aligned}
\int_{\mathbb{R}^{n}} \rho\left(\frac{\left|x^{2}\right|}{l^{2}}\right)\left|v\left(t, \theta_{-t} \omega, v_{0}\left(\theta_{-t} \omega\right)\right)\right|^{2} \mathrm{~d} x \\
\leq e^{\gamma\left(T_{1}-t\right)} \int_{\mathbb{R}^{n}} \rho\left(\frac{\left|x^{2}\right|}{l^{2}}\right)\left|v\left(T_{1}, \theta_{-t} \omega, v_{0}\left(\theta_{-t} \omega\right)\right)\right|^{2} \mathrm{~d} x \\
+\frac{c}{l} \int_{T_{1}}^{t} e^{\gamma(s-t)}\left\|v\left(s, \theta_{-t} \omega, v_{0}\left(\theta_{-t} \omega\right)\right)\right\|^{2} \mathrm{~d} s \\
+\frac{c}{l} \int_{T_{1}}^{t} e^{\gamma(s-t)}\left\|\nabla v\left(s, \theta_{-t} \omega, v_{0}\left(\theta_{-t} \omega\right)\right)\right\|^{2} \mathrm{~d} s \\
+c \cdot \int_{T_{1}}^{t} e^{\gamma(s-t)} \int_{\mathbb{R}^{n}} \rho\left(\frac{\left|x^{2}\right|}{l^{2}}\right)\left|z\left(\theta_{s-t} \omega\right)\right|^{4} \mathrm{~d} x \mathrm{~d} s \\
+\frac{\lambda^{2}+\mu^{2}}{\gamma} \int_{T_{1}}^{t} e^{\gamma(s-t)} \int_{\mathbb{R}^{n}} \rho\left(\frac{\left|x^{2}\right|}{l^{2}}\right)\left|\Delta z\left(\theta_{s-t} \omega\right)\right|^{2} \mathrm{~d} x \mathrm{~d} s .
\end{aligned}
$$

We now estimate the terms in (81) as follows.
Firstly, from (28), one deduces

$$
\begin{aligned}
\left\|v\left(T_{1}, \theta_{-t} \omega, v_{0}\left(\theta_{-t} \omega\right)\right)\right\|^{2} \leq & e^{-\gamma T_{1}}\left\|v_{0}\left(\theta_{-t} \omega\right)\right\|^{2} \\
& +\int_{0}^{T_{1}} e^{\gamma\left(\tau-T_{1}\right)} h\left(\theta_{\tau-t} \omega\right) \mathrm{d} \tau .
\end{aligned}
$$

Thus,

$$
\begin{gathered}
e^{\gamma\left(T_{1}-t\right)} \int_{\mathbb{R}^{n}} \rho\left(\frac{\left|x^{2}\right|}{l^{2}}\right)\left|v\left(T_{1}, \theta_{-t} \omega, v_{0}\left(\theta_{-t} \omega\right)\right)\right|^{2} \mathrm{~d} x \\
\leq e^{\gamma\left(T_{1}-t\right)}\left(e^{-\gamma T_{1}}\left\|v_{0}\left(\theta_{-t} \omega\right)\right\|^{2}\right. \\
\left.\quad+\int_{0}^{T_{1}} e^{\gamma\left(\tau-T_{1}\right)} h\left(\theta_{\tau-t} \omega\right) \mathrm{d} \tau\right) \\
\leq e^{-\gamma t}\left\|v_{0}\left(\theta_{-t} \omega\right)\right\|^{2}+\int_{-t}^{T_{1}-t} e^{\gamma s} h\left(\theta_{s} \omega\right) \mathrm{d} s \\
\leq e^{-\gamma t}\left\|v_{0}\left(\theta_{-t} \omega\right)\right\|^{2}+\frac{c}{2 \gamma} \cdot r(\omega) e^{(\gamma / 2)\left(T_{1}-t\right)}
\end{gathered}
$$

due to (18) and (26). Thus, for any given $\epsilon>0$, there is $T_{2}(B, \omega, \epsilon)>T_{1}$ such that, for all $t \geq T_{2}$,

$$
e^{\gamma\left(T_{1}-t\right)} \int_{\mathbb{R}^{n}} \rho\left(\frac{\left|x^{2}\right|}{l^{2}}\right)\left|v\left(T_{1}, \theta_{-t} \omega, v_{0}\left(\theta_{-t} \omega\right)\right)\right|^{2} \mathrm{~d} x \leq \epsilon .
$$

For the second, replace $T_{1}$ by $s$ in (82); then we can find that the second term at the right-hand side of (81) satisfies

$$
\begin{aligned}
& \frac{c}{l} \int_{T_{1}}^{t} e^{\gamma(s-t)}\left\|v\left(s, \theta_{-t} \omega, v_{0}\left(\theta_{-t} \omega\right)\right)\right\|^{2} \mathrm{~d} s \\
& \leq \frac{c}{l} \int_{T_{1}}^{t} e^{-\gamma t}\left\|v_{0}\left(\theta_{-t} \omega\right)\right\|^{2} \mathrm{~d} s \\
& \quad+\frac{c}{l} \int_{T_{1}}^{t} \int_{0}^{s} e^{\gamma(\tau-t)} h\left(\theta_{\tau-t} \omega\right) \mathrm{d} \tau \mathrm{d} s \\
& \leq \frac{c}{l} e^{-\gamma t}\left(t-T_{1}\right)\left\|v_{0}\left(\theta_{-t} \omega\right)\right\|^{2} \\
& \quad+\frac{c}{l} \int_{T_{1}}^{t} \int_{-t}^{s-t} e^{\gamma \tau} h\left(\theta_{\tau} \omega\right) \mathrm{d} \tau \mathrm{d} s \\
& \leq \frac{c}{l} e^{-\gamma t}\left(t-T_{1}\right)\left\|v_{0}\left(\theta_{-t} \omega\right)\right\|^{2} \\
&+\frac{c r(\omega)}{l} \int_{T_{1}}^{t} \int_{-t}^{s-t} e^{(\gamma / 2) \tau} \mathrm{d} \tau \mathrm{d} s \\
& \leq \frac{c}{l} e^{-\gamma t}\left(t-T_{1}\right)\left\|v_{0}\left(\theta_{-t} \omega\right)\right\|^{2}+\frac{c r(\omega)}{\gamma^{2} l}
\end{aligned}
$$

which implies that there exist $T_{3}(B, \omega, \epsilon)>T_{1}$ and $R_{1}(\omega, \epsilon)>$ 0 such that, for all $t \geq T_{3}$ and $l \geq R_{1}$,

$$
\frac{c}{l} \int_{T_{1}}^{t} e^{\gamma(s-t)}\left\|v\left(s, \theta_{-t} \omega, v_{0}\left(\theta_{-t} \omega\right)\right)\right\|^{2} \mathrm{~d} s \leq \epsilon .
$$


For the third, from Lemma 12, we know that there is $T_{4}(B, \omega)>T_{1}$ such that, for all $t \geq T_{4}$, the third term at the right-hand side of (81) satisfies

$$
\frac{c}{l} \int_{T_{1}}^{t} e^{\gamma(s-t)}\left\|\nabla v\left(s, \theta_{-t} \omega, v_{0}\left(\theta_{-t} \omega\right)\right)\right\|^{2} \mathrm{~d} s \leq \frac{2 c}{l \gamma} r(\omega) .
$$

Therefore, there is $R_{2}(\omega, \epsilon)>0$ such that, for all $t \geq T_{4}$ and $l \geq R_{2}$

$$
\frac{c}{l} \int_{T_{1}}^{t} e^{\gamma(s-t)}\left\|\nabla v\left(s, \theta_{-t} \omega, v_{0}\left(\theta_{-t} \omega\right)\right)\right\|^{2} \mathrm{~d} s \leq \epsilon .
$$
by

Finally, note that the last two terms in (81) can be bounded

$$
\begin{aligned}
& c \cdot \int_{T_{1}}^{t} e^{\gamma(s-t)} \\
& \quad \times \int_{\mathbb{R}^{n}} \rho\left(\frac{\left|x^{2}\right|}{l^{2}}\right)\left(\left|\Delta z\left(\theta_{s-t} \omega\right)\right|^{2}+\left|z\left(\theta_{s-t} \omega\right)\right|^{4}\right) \mathrm{d} x \mathrm{~d} s
\end{aligned}
$$

and $z\left(\theta_{t} \omega\right)=\sum_{j=1}^{m} \varphi_{j} z_{j}\left(\theta_{t} \omega_{j}\right)$, where $\varphi_{j} \in H^{2}\left(\mathbb{R}^{n}\right) \cap$ $W^{2,4}\left(\mathbb{R}^{n}\right)$, and we can find $R_{3}(\omega, \epsilon)>0$ such that, for all $l \geq R_{3}$ and $j=1,2, \ldots, m$,

$$
\begin{aligned}
& \int_{|x| \geq l}\left(\left|\varphi_{j}(x)\right|^{2}+\left|\varphi_{j}(x)\right|^{4}+\left|\Delta \varphi_{j}(x)\right|^{2}\right) \mathrm{d} x \\
& \leq \min \left\{\frac{\gamma \epsilon}{m^{4} c r(\omega)}, \frac{\epsilon}{2 m r(\omega)}\right\} .
\end{aligned}
$$

Accordingly, we have the following estimates for the last two terms in (81):

$$
\begin{aligned}
& c \cdot \int_{T_{1}}^{t} e^{\gamma(s-t)} \int_{\mathbb{R}^{n}} \rho\left(\frac{\left|x^{2}\right|}{l^{2}}\right)\left|z\left(\theta_{s-t} \omega\right)\right|^{4} \mathrm{~d} x \mathrm{~d} s \\
& +\frac{\lambda^{2}+\mu^{2}}{\gamma} \int_{T_{1}}^{t} e^{\gamma(s-t)} \\
& \quad \times \int_{\mathbb{R}^{n}} \rho\left(\frac{\left|x^{2}\right|}{l^{2}}\right)\left|\Delta z\left(\theta_{s-t} \omega\right)\right|^{2} \mathrm{~d} x \mathrm{~d} s \\
& \leq c \cdot \int_{T_{1}}^{t} e^{\gamma(s-t)} \\
& \times \int_{\mathbb{R}^{n}} \rho\left(\frac{\left|x^{2}\right|}{l^{2}}\right) \\
& \leq c \cdot \int_{T_{1}}^{t} e^{\gamma(s-t)} \times\left(\left|\Delta z\left(\theta_{s-t} \omega\right)\right|^{2}+\left|z\left(\theta_{s-t} \omega\right)\right|^{4}\right) \mathrm{d} x \mathrm{~d} s \\
& \times \int_{|x| \geq l}\left(\left|\Delta z\left(\theta_{s-t} \omega\right)\right|^{2}+\left|z\left(\theta_{s-t} \omega\right)\right|^{4}\right) \mathrm{d} x \mathrm{~d} s \\
& \left.\leq c m^{4} \cdot \int_{T_{1}}^{t} e^{\gamma(s-t)}+\left|\varphi_{j}\right|^{4}\left|z_{j}\left(\theta_{s-t} \omega_{j}\right)\right|^{4}\right) \mathrm{d} x \mathrm{~d} s \\
& \times \sum_{j=1}^{m} \int_{|x| \geq l}\left(\left|\Delta \varphi_{j}\right|^{2}\left|z_{j}\left(\theta_{s-t} \omega_{j}\right)\right|^{2}\right. \\
& \leq
\end{aligned}
$$

$$
\begin{aligned}
& \leq \frac{\gamma \epsilon}{r(\omega)} \int_{T_{1}}^{t} e^{\gamma(s-t)} \\
& \quad \times \sum_{j=1}^{m}\left(\left|z_{j}\left(\theta_{s-t} \omega_{j}\right)\right|^{2}+\left|z_{j}\left(\theta_{s-t} \omega_{j}\right)\right|^{4}\right) \mathrm{d} s
\end{aligned}
$$$$
\leq \frac{\gamma \epsilon}{r(\omega)} \int_{T_{1}}^{t} e^{\gamma(s-t)} h\left(\theta_{s-t} \omega\right) \mathrm{d} s
$$$$
\leq \frac{\gamma \epsilon}{r(\omega)} \int_{T_{1}-t}^{0} e^{\gamma \tau} h\left(\theta_{\tau} \omega\right) \mathrm{d} \tau
$$$$
\leq \frac{\gamma \epsilon}{r(\omega)} \int_{T_{1}-t}^{0} e^{(\gamma / 2) \tau} \mathrm{d} \tau \leq \epsilon .
$$

Let $T^{*}=T(B, \omega, \epsilon)=\max \left\{T_{1}, T_{2}, T_{3}, T_{4}\right\}$ and $R^{*}=R(\omega, \epsilon)=$ $\max \left\{R_{1}, R_{2}, R_{3}\right\}$. Then from (81), (84), (86), (88), and (91), we know that, for all $t \geq T^{*}$ and $l \geq R^{*}$,

$$
\int_{\mathbb{R}^{n}} \rho\left(\frac{\left|x^{2}\right|}{l^{2}}\right)\left|v\left(t, \theta_{-t} \omega, v_{0}\left(\theta_{-t} \omega\right)\right)\right|^{2} \mathrm{~d} x \leq 4 \epsilon .
$$

That is, for any $t \geq T^{*}$ and $l \geq R^{*}$,

$$
\begin{aligned}
& \int_{|x| \geq l}\left|v\left(t, \theta_{-t} \omega, v_{0}\left(\theta_{-t} \omega\right)\right)\right|^{2} \mathrm{~d} x \\
& \leq \int_{\mathbb{R}^{n}} \rho\left(\frac{\left|x^{2}\right|}{l^{2}}\right)\left|v\left(t, \theta_{-t} \omega, v_{0}\left(\theta_{-t} \omega\right)\right)\right|^{2} \mathrm{~d} x \leq 4 \epsilon .
\end{aligned}
$$

The proof is completed.

Lemma 17. Suppose $\sqrt{3} \kappa \geq|\beta|$, and let $B=\{B(\omega)\}_{\omega \in \Omega} \in \mathscr{D}$ and $u_{0}(\omega) \in B(\omega)$; then, for every $\epsilon>0$ and $P$-a.e. $\omega \in \Omega$, there exist $T^{*}=T(B, \omega, \epsilon)>0$ and $R^{*}=R^{*}(\omega, \epsilon)$ such that the solution $u\left(t, \omega, u_{0}(\omega)\right)$ of (1)-(2) satisfies, for all $t \geq T^{*}$,

$$
\int_{|x| \geq R^{*}}\left|u\left(t, \theta_{-t} \omega, u_{0}\left(\theta_{-t} \omega\right)\right)(x)\right|^{2} d x \leq \epsilon .
$$

Proof. Let $T^{*}$ and $R^{*}$ be the constants in Lemma 16. Then due to (16) and (90) we know that, for all $t \geq T^{*}$ and $l \geq R^{*}$,

$$
\begin{aligned}
\int_{|x| \geq R^{*}}|z(\omega)|^{2} \mathrm{~d} x & =\int_{|x| \geq R^{*}}\left|\sum_{j=1}^{m} \varphi_{j} z_{j}\left(\omega_{j}\right)\right|^{2} \mathrm{~d} x \\
& \leq m \int_{|x| \geq R^{*}} \sum_{j=1}^{m}\left|\varphi_{j}\right|^{2}\left|z_{j}\left(\omega_{j}\right)\right|^{2} \mathrm{~d} x \\
& \leq \frac{\epsilon}{2 r(\omega)} \sum_{j=1}^{m}\left|z_{j}\left(\omega_{j}\right)\right|^{2} \leq \frac{\epsilon}{2} .
\end{aligned}
$$


Thus, together with Lemma 16, we derive, for all $t \geq T^{*}$ and $l \geq R^{*}$,

$$
\begin{aligned}
& \int_{|x| \geq R^{*}}\left|u\left(t, \theta_{-t} \omega, u_{0}\left(\theta_{-t} \omega\right)\right)(x)\right|^{2} \mathrm{~d} x \\
& =\int_{|x| \geq R^{*}}\left|v\left(t, \theta_{-t} \omega, v_{0}\left(\theta_{-t} \omega\right)\right)(x)+z(\omega)\right|^{2} \mathrm{~d} x \\
& \leq 2 \int_{|x| \geq R^{*}}\left|v\left(t, \theta_{-t} \omega, v_{0}\left(\theta_{-t} \omega\right)\right)(x)\right|^{2} \\
& \quad+2 \int_{|x| \geq R^{*}}|z(\omega)|^{2} \mathrm{~d} x \\
& \leq 3 \epsilon .
\end{aligned}
$$

The proof is completed.

Up to now, we are ready to give the $\mathscr{D}$-pullback asymptotic compactness of $\phi$, based on the former uniform estimates referring to the tails of solutions.

Proposition 18. Suppose that $\sqrt{3} \kappa \geq|\beta|$, and then the random dynamical system $\phi$ is $\mathscr{D}$-pullback asymptotically compact in $\mathbb{L}^{2}\left(\mathbb{R}^{n}\right)$. That is to say, for P-a.e. $\omega \in \Omega$, the sequence $\left\{\phi\left(t_{n}, \theta_{-t_{n}} \omega, u_{0, n}\left(\theta_{-t_{n}} \omega\right)\right)\right\}_{n=1}^{\infty}$ has a convergent subsequence in $\mathbb{L}^{2}\left(\mathbb{R}^{n}\right)$ for $t_{n} \longrightarrow \infty, B=\{B(\omega)\}_{\omega \in \Omega} \in \mathscr{D}$, and $u_{0, n}\left(\theta_{-t_{n}} \omega\right) \in$ $B\left(\theta_{-t_{n}} \omega\right)$.

Proof. Let $t_{n} \longrightarrow \infty, B=\{B(\omega)\}_{\omega \in \Omega} \in \mathscr{D}$, and $u_{0, n}\left(\theta_{-t_{n}} \omega\right) \epsilon$ $B\left(\theta_{-t_{n}} \omega\right)$. By Proposition 11, we know that, for $P$-a.e. $\omega \in \Omega$,

$$
\left\{\phi\left(t_{n}, \theta_{-t_{n}} \omega, u_{0, n}\left(\theta_{-t_{n}} \omega\right)\right)\right\}_{n=1}^{\infty} \quad \text { is bounded in } \mathbb{L}^{2}\left(\mathbb{R}^{n}\right) .
$$

So, there is $\xi \in \mathbb{L}^{2}\left(\mathbb{R}^{n}\right)$ such that, up to a subsequence,

$$
\phi\left(t_{n}, \theta_{-t_{n}} \omega, u_{0, n}\left(\theta_{-t_{n}} \omega\right)\right) \longrightarrow \xi \quad \text { weakly in } \mathbb{L}^{2}\left(\mathbb{R}^{n}\right) \text {. }
$$

It only remains to prove that the weak convergence of (98) is indeed strong convergence. Let $\epsilon>0$ be small enough. Since $\xi \in \mathbb{L}^{2}\left(\mathbb{R}^{n}\right)$, there exists $R_{1}=R(\epsilon)>0$, such that

$$
\int_{|x| \geq R_{1}}|\xi(x)|^{2} \mathrm{~d} x \leq \epsilon .
$$

From Lemma 17, there are $T_{1}(B, \omega, \epsilon)$ and $R_{2}(\omega, \epsilon)>R_{1}(\epsilon)>$ 0 , for $P$-a.e. $\omega \in \Omega$, such that, for all $t \geq T_{1}$,

$$
\int_{|x| \geq R_{2}}\left|\phi\left(t, \theta_{-t} \omega, u_{0}\left(\theta_{-t} \omega\right)\right)\right|^{2} \mathrm{~d} x \leq \epsilon .
$$

Since $t_{n} \longrightarrow \infty$, let $N_{1}=N_{1}(B, \omega, \epsilon)$ be large enough such that $t_{n} \geq T_{1}$ for every $n \geq N_{1}$. Hence, it follows from (100) that, for all $n \geq N_{1}$,

$$
\int_{|x| \geq R_{2}}\left|\phi\left(t_{n}, \theta_{-t_{n}} \omega, u_{0, n}\left(\theta_{-t_{n}} \omega\right)\right)\right|^{2} \mathrm{~d} x \leq \epsilon .
$$

On the other hand, from Proposition 11 and Lemma 15, there is $T_{2}=T_{2}(B, \omega)$ such that, for all $t \geq T_{2}$,

$$
\left\|\phi\left(t, \theta_{-t} \omega, u_{0}\left(\theta_{-t} \omega\right)\right)\right\|_{H^{1}\left(\mathbb{R}^{n}\right)}^{2} \leq \frac{4 c}{\gamma} \cdot e^{\gamma} \cdot r(\omega) .
$$

Let $N_{2}=N_{2}(B, \omega)>N_{1}$ such that $t_{n} \geq T_{2}$ for $n \geq N_{2}$. Thus, from (102), we know that, for all $n \geq N_{2}$,

$$
\left\|\phi\left(t_{n}, \theta_{-t_{n}} \omega, u_{0, n}\left(\theta_{-t_{n}} \omega\right)\right)\right\|_{H^{1}\left(\mathbb{R}^{n}\right)}^{2} \leq \frac{4 c}{\gamma} \cdot e^{\gamma} \cdot r(\omega) .
$$

Denote $Q_{R_{2}}$ for the set $\left\{x \in \mathbb{R}^{n}:|x| \leq R_{2}\right\}$. Due to the compactness of embedding $H^{1}\left(Q_{R_{2}}\right) \hookrightarrow \mathbb{L}^{2}\left(Q_{R_{2}}\right)$, we deduce from (103) that, up to a subsequence,

$$
\phi\left(t_{n}, \theta_{-t_{n}} \omega, u_{0, n}\left(\theta_{-t_{n}} \omega\right)\right) \longrightarrow \xi \text { strongly in } \mathbb{L}^{2}\left(Q_{R_{2}}\right),
$$

which tells us that, for the given $\epsilon>0$, there exists $N_{3}=$ $N_{3}(B, \omega, \epsilon)>N_{2}$ such that, for all $n \geq N_{3}$,

$$
\left\|\phi\left(t_{n}, \theta_{-t_{n}} \omega, u_{0, n}\left(\theta_{-t_{n}} \omega\right)\right)-\xi\right\|_{\mathbb{L}^{2}\left(Q_{R_{2}}\right)}^{2} \leq \epsilon .
$$

By (99), (101), and (105), we conclude that, for all $n \geq N_{3}$,

$$
\begin{aligned}
& \left\|\phi\left(t_{n}, \theta_{-t_{n}} \omega, u_{0, n}\left(\theta_{-t_{n}} \omega\right)\right)-\xi\right\|_{\mathbb{L}^{2}\left(\mathbb{R}^{n}\right)}^{2} \\
& \leq \int_{|x| \geq R_{2}}\left|\phi\left(t_{n}, \theta_{-t_{n}} \omega, u_{0, n}\left(\theta_{-t_{n}} \omega\right)\right)-\xi\right|^{2} \mathrm{~d} x \\
& \quad+\int_{|x| \leq R_{2}}\left|\phi\left(t_{n}, \theta_{-t_{n}} \omega, u_{0, n}\left(\theta_{-t_{n}} \omega\right)\right)-\xi\right|^{2} \mathrm{~d} x \leq 5 \epsilon .
\end{aligned}
$$

Therefore, up to a subsequence,

$$
\phi\left(t_{n}, \theta_{-t_{n}} \omega, u_{0, n}\left(\theta_{-t_{n}} \omega\right)\right) \longrightarrow \xi \quad \text { strongly in } \mathbb{L}^{2}\left(\mathbb{R}^{n}\right)
$$

is verified.

Up to now, we have proved that $\phi$ has a closed random absorbing set $\{K(\omega)\}_{\omega \in \Omega}$ in $\mathscr{D}$ by Proposition 11 and is $\mathscr{D}$ pullback asymptotically compact in $\mathbb{L}^{2}\left(\mathbb{R}^{n}\right)$, which is present in Proposition 18. So, the existence of unique $\mathscr{D}$-random attractor for $\phi$ stated in Theorem 1 immediately follows from Proposition 9.

\section{Conflict of Interests}

The authors declare that there is no conflict of interests regarding the publication of this paper.

\section{Acknowledgments}

This work is supported by the project sponsored by SRF for ROCS, SEM (no. 114329A4C11604), National Natural Science Foundation of China (nos. 11101370, 11101283, 11001041, and 11211130093), and the "521" Talent Program of Zhejiang SciTech University (ZSTU) under Grant no. 11430132521304. 


\section{References}

[1] A. V. Babin and M. I. Vishik, Attractors of Evolution Equations, North Holland, 1992.

[2] X.-C. Fu and J. Duan, "Global attractors and invariant measures for non-invertible planar piecewise isometric maps," Physics Letters A: General, Atomic and Solid State Physics, vol. 371, no. 4, pp. 285-290, 2007.

[3] X.-C. Fu and J. Duan, "On global attractors for a class of nonhyperbolic piecewise affine maps," Physica D: Nonlinear Phenomena, vol. 237, no. 24, pp. 3369-3376, 2008.

[4] J. K. Hale, "Asymptotic behavior of dissipative systems," American Mathematical Society, vol. 15, 1988.

[5] J. C. Robinson, Infinite-Dimensional Dynamical Systems, Cambridge University Press, Cambridge, UK, 2001.

[6] G. R. Sell and Y. You, Dynamics of Evolutionary Equations, Springer, New York, NY, USA, 2002.

[7] R. Temam, Infinite Dimensional Dynamical Systems in Mechanics and Physics, Springer, New York, NY, USA, 2nd edition, 1997.

[8] H. Crauel, A. Debussche, and F. Flandoli, "Random attractors," Journal of Dynamics and Differential Equations, vol. 9, no. 2, pp. 307-341, 1997.

[9] H. Crauel and F. Flandoli, "Attractors for random dynamical systems," Probability Theory and Related Fields, vol. 100, no. 3, pp. 365-393, 1994.

[10] F. Flandoli and B. Schmalfuss, "Random attractors for the 3 D stochastic navier-stokes equation with multiplicative white noise," Stochastics and Stochastic Reports, vol. 59, pp. 21-45, 1996.

[11] G. Wang, B. Guo, and Y. Li, "The asymptotic behavior of the stochastic Ginzburg-Landau equation with additive noise," Applied Mathematics and Computation, vol. 198, no. 2, pp. 849857, 2008.

[12] Y. Desheng, "The asymptotic behavior of the stochastic Ginzburg-Landau equation with multiplicative noise," Journal of Mathematical Physics, vol. 45, no. 11, pp. 4064-4076, 2004.

[13] J. M. Ball, "Continuity properties and global attractors of generalized semiflows and the Navier-Stokes equations," Journal of Nonlinear Science, vol. 7, no. 2, pp. 475-502, 1997.

[14] J. M. Ball, "Global attractors for damped semilinear wave equations," Discrete and Continuous Dynamical Systems, vol. 10, no. 1-2, pp. 31-52, 2004.

[15] T. Caraballo, G. Łukaszewicz, and J. Real, "Pullback attractors for asymptotically compact non-autonomous dynamical systems," Nonlinear Analysis: Theory, Methods and Applications, vol. 64, no. 3, pp. 484-498, 2006.

[16] J. M. Ghidaglia, "A note on the strong convergence towards attractors of damped forced KdV equations," Journal of Differential Equations, vol. 110, no. 2, pp. 356-359, 1994.

[17] O. Goubet and R. M. S. Rosa, "Asymptotic smoothing and the global attractor of a weakly damped $\mathrm{KdV}$ equation on the real line," Journal of Differential Equations, vol. 185, no. 1, pp. 25-53, 2002.

[18] J. Ning, "The H1-compact global attractor for the solutions to the Navier-Stokes equations in two-dimensional unbounded domains," Nonlinearity, vol. 13, no. 4, pp. 1227-1238, 2000.

[19] I. Moise and R. Rosa, "On the regularity of the global attractor of a weakly damped, forced Korteweg-de Vries equation," Advances in Differential Equations, vol. 2, pp. 257-296, 1997.

[20] I. Moise, R. Rosa, and X. Wang, "Attractors for non-compact semigroups via energy equations," Nonlinearity, vol. 11, no. 5, pp. 1369-1393, 1998.
[21] R. Rosa, "The global attractor for the $2 \mathrm{D}$ navier-stokes flow on some unbounded domains," Nonlinear Analysis: Theory, Methods and Applications, vol. 32, no. 1, pp. 71-85, 1998.

[22] X. Wang, "An energy equation for the weakly damped driven nonlinear Schrödinger equations and its application to their attractors," Physica D: Nonlinear Phenomena, vol. 88, no. 3-4, pp. 167-175, 1995.

[23] P. W. Bates, K. Lu, and B. Wang, "Random attractors for stochastic reaction-diffusion equations on unbounded domains," Journal of Differential Equations, vol. 246, no. 2, pp. 845-869, 2009.

[24] L. Arnold, Random Dynamical Systems, Springer, 1998.

[25] P. W. Bates, H. Lisei, and K. Lu, "Attractors for stochastic lattice dynamical systems," Stochastics and Dynamics, vol. 6, no. 1, pp. $1-21,2006$.

[26] H. Crauel, "Random point attractors versus random set attractors," Journal of the London Mathematical Society, vol. 63, no. 2, pp. 413-427, 2001. 


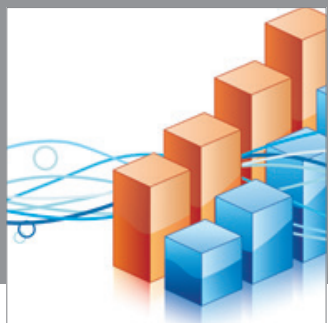

Advances in

Operations Research

mansans

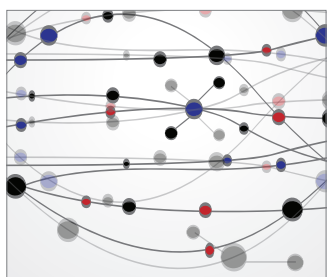

The Scientific World Journal
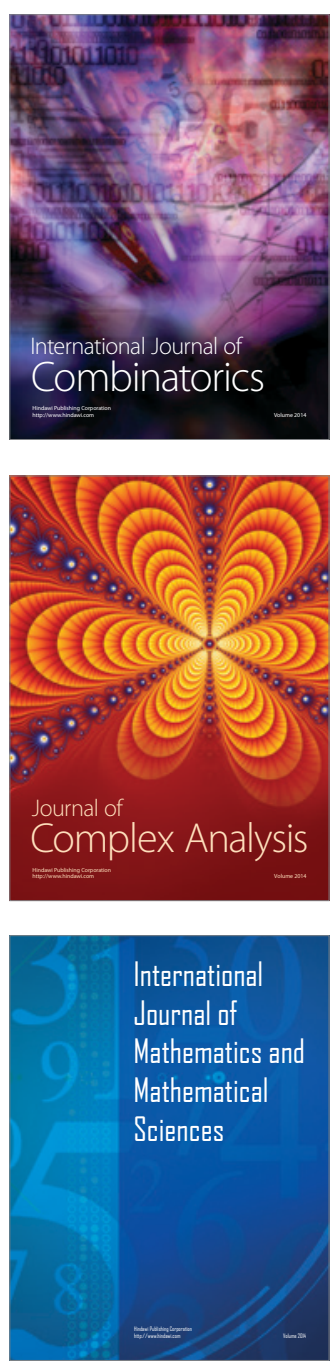
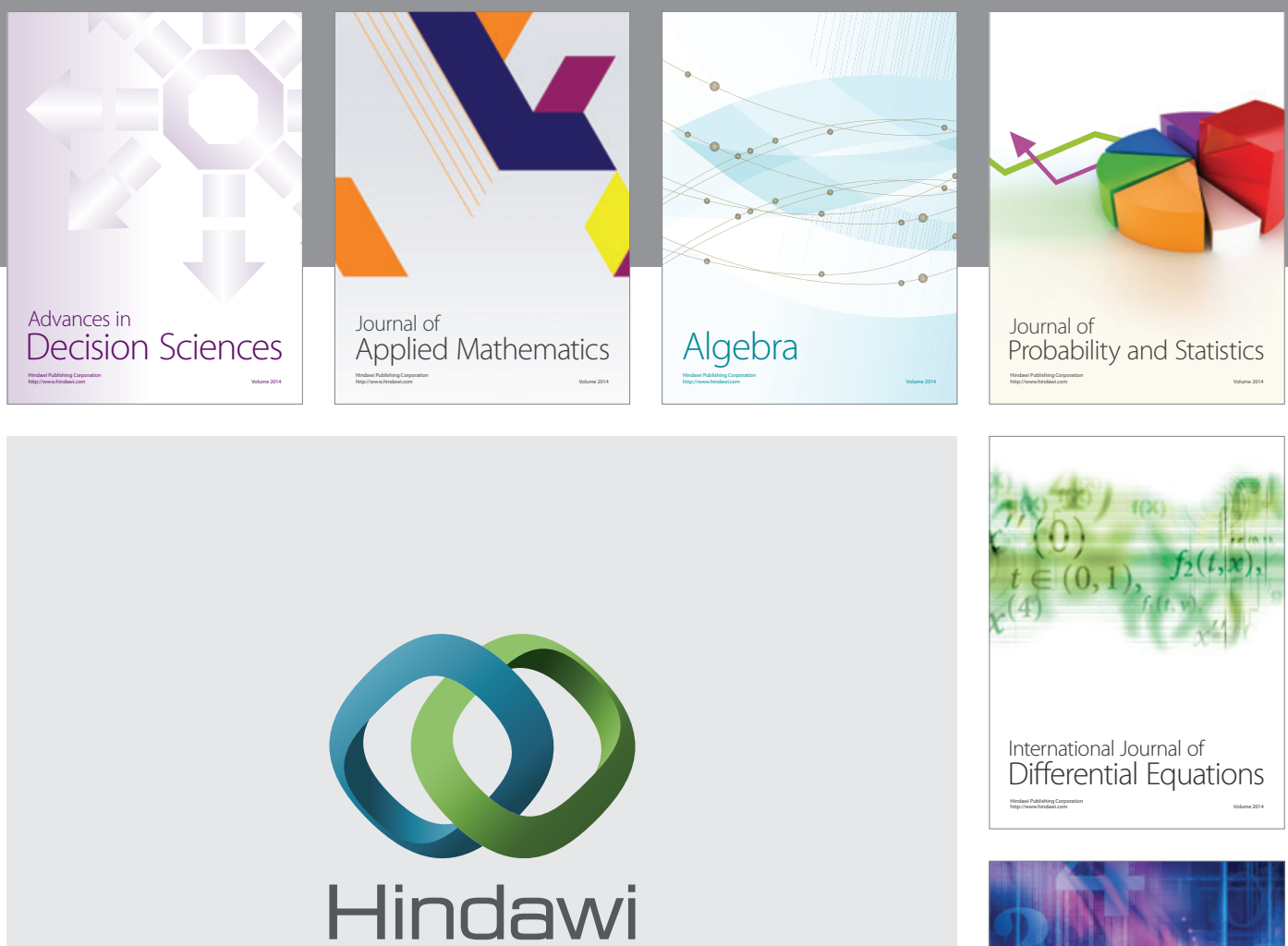

Submit your manuscripts at http://www.hindawi.com
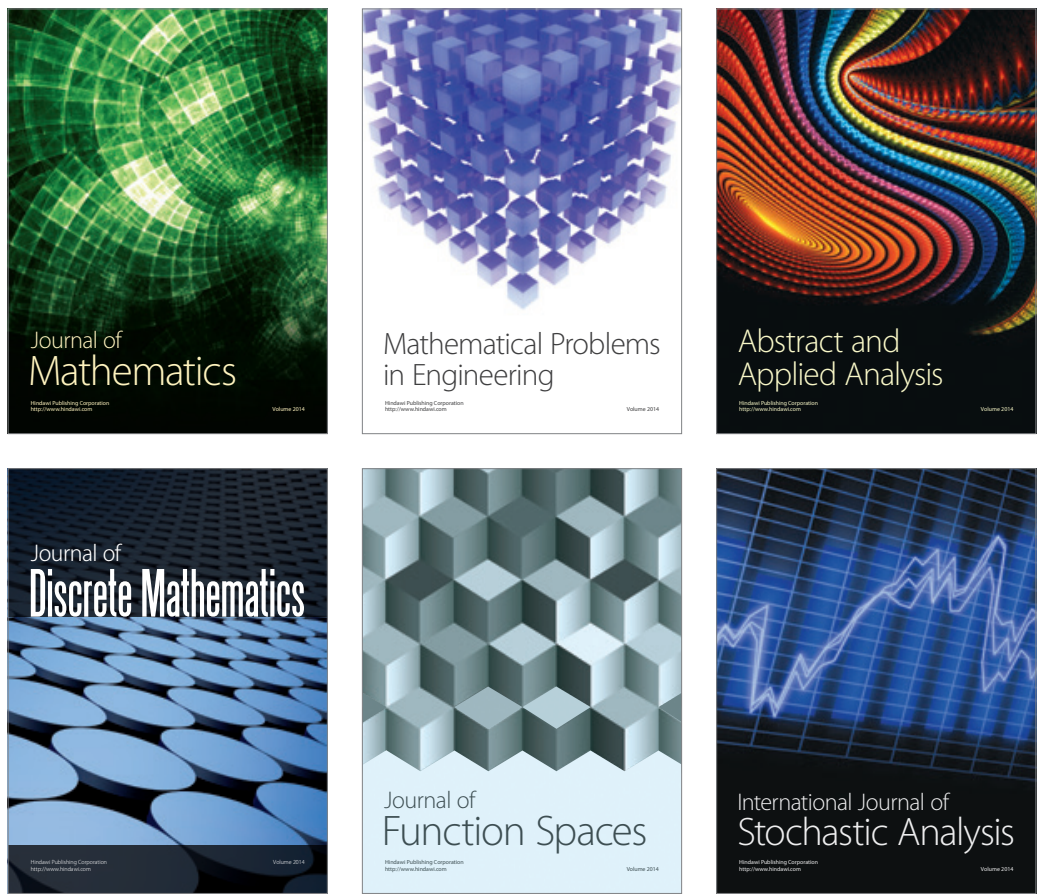

Journal of

Function Spaces

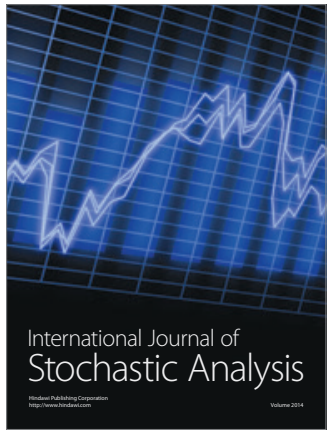

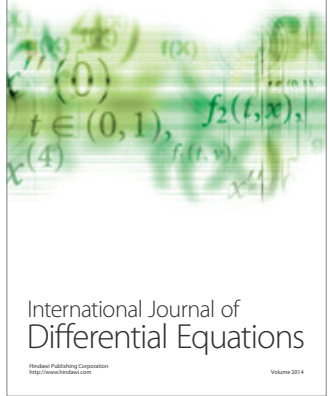
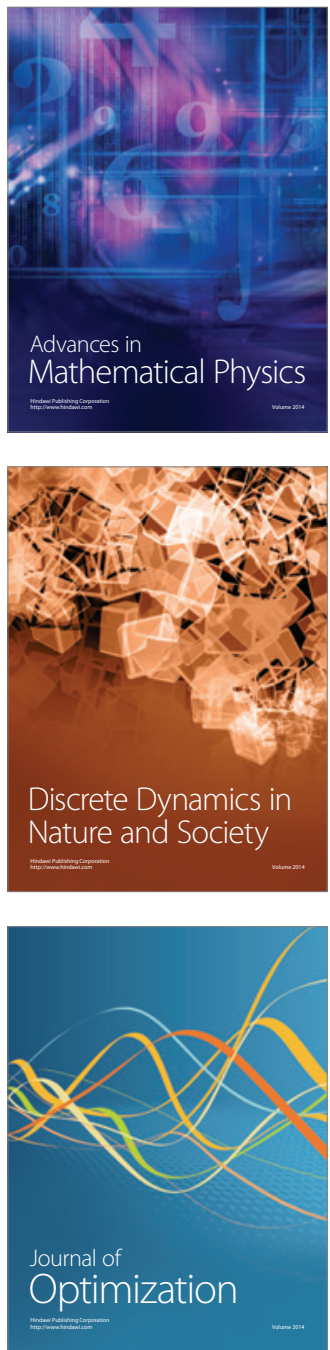